\title{
Existence of multiple positive solutions for $p$-Laplacian multipoint boundary value problems on time scales
}

\section{Abdulkadir Dogan*}

\section{"Correspondence:}

abdulkadir.dogan@agu.edu.t

Department of Applied

Mathematics, Faculty of Computer

Sciences, Abdullah Gul University,

Kayseri, 38039, Turkey

\begin{abstract}
In this paper, we consider $p$-Laplacian multipoint boundary value problems on time scales. By using a generalization of the Leggett-Williams fixed point theorem due to Bai and Ge, we prove that a boundary value problem has at least three positive solutions. Moreover, we study existence of positive solutions of a multipoint boundary value problem for an increasing homeomorphism and homomorphism on time scales. By using fixed point index theory, sufficient conditions for the existence of at least two positive solutions are provided. Examples are given to illustrate the results.
\end{abstract} MSC: 34B15; 34B16; 34B18; 39A10

Keywords: time scales; boundary value problem; $p$-Laplacian; positive solutions; fixed point theorem

\section{Introduction}

The theory of dynamic equation on time scales (or measure chains) was initiated by Stefan Hilger in his PhD thesis in 1988 [1] (supervised by Bernd Aulbach) as a means of unifying structure for the study of differential equations in the continuous case and study of finite difference equations in the discrete case. In recent years, it has gained a considerable amount of interest and attracted the attention of many researchers, see, for example, [2-13]. It is still a new area, and the research in this area is rapidly growing. The study of time scales has led to several important applications, e.g., in the study of insect population models, heat transfer, neural networks, phytoremediation of metals, wound healing, and epidemic models.

p-Laplacian equations for boundary value problems (BVPs) with nonlinearity depending on the first order derivative have been studied extensively, see [14-20] and references therein. However, there are few papers concerning $p$-Laplacian equations with nonlinearity depending on the first order derivative for BVPs on time scales, see [5, 7].

In this paper, we study the following three boundary value problems on time scales.

(1) We are interested in the existence of at least three positive solutions to the following $p$-Laplacian multipoint BVP on time scales

$$
\begin{aligned}
& \left(\phi_{p}\left(u^{\Delta}(t)\right)\right)^{\Delta}+q(t) f\left(t, u(t), u^{\Delta}(t)\right)=0, \quad t \in(0, T)_{\mathbb{T}}, \\
& u(0)=\sum_{i=1}^{n-2} \alpha_{i} u\left(\xi_{i}\right), \quad \phi_{p}\left(u^{\Delta}(T)\right)=\sum_{i=1}^{n-2} \beta_{i} \phi_{p}\left(u^{\Delta}\left(\xi_{i}\right)\right),
\end{aligned}
$$

2013 Dogan; licensee Springer. This is an Open Access article distributed under the terms of the Creative Commons Attribution License (http://creativecommons.org/licenses/by/2.0), which permits unrestricted use, distribution, and reproduction in any medium, provided the original work is properly cited. 
where $\phi_{p}(u)$ is a $p$-Laplacian operator, i.e., $\phi_{p}(u)=|u|^{p-2} u$, for $p>1$, with $\left(\phi_{p}\right)^{-1}=\phi_{q}$ and $\frac{1}{p}+\frac{1}{q}=1$. The usual notation and terminology for time scales as can be found in [21, 22], will be used here. The interesting point is that the nonlinear term $f$ is involved with the first order derivative explicitly and the main tool is a fixed point theorem due to Bai and Ge. The results are even new for the special cases of difference equations and differential equations, as well as in the general time scale setting.

The present work is motivated by the recent papers [5,15]. In [15], Yang and Xiao studied the existence of multiple positive solutions for $\phi$-Laplacian multipoint BVPs

$$
\begin{aligned}
& \left(\phi\left(x^{\prime}(t)\right)\right)^{\prime}+q(t) f\left(t, x(t), x^{\prime}(t)\right)=0, \quad t \in(0,1), \\
& x(0)=\sum_{i=1}^{n-2} \alpha_{i} x\left(\xi_{i}\right), \quad \phi\left(x^{\prime}(1)\right)=\sum_{i=1}^{n-2} \beta_{i} \phi\left(x^{\prime}\left(\xi_{i}\right)\right),
\end{aligned}
$$

where $\phi(\cdot)$ is an odd and increasing homeomorphism, $\xi_{i} \in(0,1)$ with $0<\xi_{1}<\xi_{2}<\cdots<$ $\xi_{n-2}<1, \alpha_{i}$ and $\beta_{i}$ are nonnegative constants and $f\left(t, x(t), x^{\prime}(t)\right)$ is continuous and allowed to change sign.

(2) We consider the existence of at least three positive solutions to the following $p$-Laplacian multipoint boundary value problem (BVP) on time scales

$$
\begin{aligned}
& \left(\phi_{p}\left(u^{\Delta}(t)\right)\right)^{\nabla}+a(t) f\left(t, u(t), u^{\Delta}(t)\right)=0, \quad t \in(0, T)_{\mathbb{T}}, \\
& \phi_{p}\left(u^{\Delta}(0)\right)=\sum_{i=1}^{m-2} a_{i} \phi_{p}\left(u^{\Delta}\left(\xi_{i}\right)\right), \quad u(T)=\sum_{i=1}^{m-2} b_{i} u\left(\xi_{i}\right),
\end{aligned}
$$

where $\phi_{p}(u)$ is $p$-Laplacian operator, i.e., $\phi_{p}(u)=|u|^{p-2} u$, for $p>1$, with $\left(\phi_{p}\right)^{-1}=\phi_{q}$ and $1 / p+1 / q=1$.

Recently, much attention has been focused on the study of multipoint positive solutions of BVPs on time scales. When the nonlinear term $f$ does not depend on the first order derivative, many researchers study multipoint boundary conditions on time scales, see $[11,12,23-26]$. However, little work has been done on the existence of positive solutions for multipoint BVP on time scales when the nonlinear term is involved in the first order derivative explicitly, see [5].

In recent papers, the authors in $[6,24]$ have investigated the existence of positive solutions of the following BVP on time scales:

$$
\begin{array}{ll}
\left(\phi\left(u^{\Delta}(t)\right)\right)^{\nabla}+a(t) f(t, u(t))=0, & t \in[0, T], \\
\phi\left(u^{\Delta}(0)\right)=\sum_{i=1}^{m-2} a_{i} \phi\left(u^{\Delta}\left(\xi_{i}\right)\right), & u(T)=\sum_{i=1}^{m-2} b_{i} u\left(\xi_{i}\right),
\end{array}
$$

where $\phi: R \rightarrow R$ is an increasing homeomorphism and a homomorphism and $\phi(0)=0$ and

$$
\begin{aligned}
& \left(\phi\left(u^{\Delta}(t)\right)\right)^{\nabla}+a(t) f(t, u(t))=0, \quad t \in(0, T), \\
& u(0)=\sum_{i=1}^{m-2} a_{i} u\left(\xi_{i}\right), \quad \phi\left(u^{\Delta}(T)\right)=\sum_{i=1}^{m-2} b_{i} \phi\left(u^{\Delta}\left(\xi_{i}\right)\right),
\end{aligned}
$$


where $\phi: R \rightarrow R$ is an increasing homeomorphism and a positive homomorphism and $\phi(0)=0$.

All the above-mentioned works about positive solutions were done under the assumption that $f$ is allowed to depend just on $u$, while the first order derivative $u^{\Delta}$ is not involved explicitly in the nonlinear $\operatorname{term} f$.

Motivated by all the works above, our main results will depend on an application of a generalization of the Leggett-Williams fixed point theorem due to Bai and Ge. Here, the emphasis is that the nonlinear term is involved explicitly with the first order derivative. We shall prove that the BVP (1.3) and (1.4) has at least three positive solutions.

(3) We will be concerned with proving the existence of positive solutions to the boundary value problem on time scale $\mathbb{T}$ given by

$$
\begin{aligned}
& \left(\phi\left(u^{\Delta}(t)\right)\right)^{\nabla}+q(t) f(t, u(t))=0, \quad t \in(0, T)_{\mathbb{T}}, \\
& \phi\left(u^{\Delta}(0)\right)=0, \quad u(T)=\sum_{i=1}^{m-2} a_{i} u\left(\xi_{i}\right),
\end{aligned}
$$

where $\phi: R \rightarrow R$ is an increasing homeomorphism and a homomorphism and $\phi(0)=0$.

A projection $\phi: R \rightarrow R$ is called an increasing homeomorphism and a homomorphism if the following conditions are satisfied:

(i) if $x \leq y$, then $\phi(x) \leq \phi(y), \forall x, y \in R$;

(ii) $\phi$ is a continuous bijection and its inverse mapping is also continuous;

(iii) $\phi(x y)=\phi(x) \phi(y), \forall x, y \in R$.

In recent years, much attention has been paid to the existence of positive solutions for nonlinear boundary value problems on time scales, see [11-13, 26-29] and the reference therein. On the other hand, multipoint nonlinear boundary value problems with $p$-Laplacian operators on time scales have also been studied extensively in the literature $[26,30-33]$. However, to the best of our knowledge, there are few works on the increasing homeomorphism and homomorphism on time scales [34].

$\mathrm{Su}$ et al. [26] considered the following $m$-point singular $p$-Laplacian boundary value problem on time scales of the form

$$
\begin{aligned}
& {\left[\varphi_{p}\left(u^{\Delta}(t)\right)\right]^{\nabla}+q(t) f(t, u(t))=0, \quad t \in(0, T)_{\mathbb{T}},} \\
& u(0)=\sum_{i=1}^{m-2} \alpha_{i} u\left(\xi_{i}\right), \quad u^{\Delta}(T)-\sum_{i=1}^{m-2} \psi_{i}\left(u\left(\xi_{i}\right)\right)=0,
\end{aligned}
$$

where $\varphi_{p}(u)=|u|^{p-2} u$, for $p>1 . \psi_{i}: \mathbb{R} \rightarrow \mathbb{R}$ is continuous and nondecreasing $0<\xi_{1}<\xi_{2}<$ $\cdots<\xi_{m-2}<\rho(T)$. By using the well-known Schauder fixed point theorem and upper and lower solution method, they obtained some new existence criteria for positive solutions of the boundary value problem.

In [34], Liang and Zhang studied the existence of positive solutions of boundary value problems on time scales:

$$
\begin{aligned}
& {\left[\varphi\left(u^{\Delta}(t)\right)\right]^{\nabla}+a(t) f(u(t))=0, \quad t \in[0, T]_{\mathbb{T}},} \\
& u(0)=\sum_{i=1}^{m-2} \alpha_{i} u\left(\xi_{i}\right), \quad u^{\Delta}(T)=0,
\end{aligned}
$$


where $\varphi: \mathbb{R} \rightarrow \mathbb{R}$ is an increasing homeomorphism and a positive homomorphism and $\varphi(0)=0$. They obtained the countably many positive solutions by using a fixed point index theory and fixed point theorem.

This work is motivated by recent papers [26, 34]. Existence of at least two positive solutions to BVP (1.5) and (1.6) are established by means of fixed point index theory. We also point out that when $\mathbb{T}=R, p=2$, (1.5) and (1.6) becomes a boundary value problem of differential equations and is just the problem considered in [35]. Our main results improve and extend the main results of $[35,36]$.

\section{Preliminaries}

In this section, we provide some background materials from the theory of cones in Banach spaces. The following definitions can be found in the book by Deimling [37], as well as in the book by Guo and Lakshmikantham [38].

Definition 2.1 Let $E$ be a real Banach space. A nonempty, closed, convex set $P \subset E$ is a cone if it satisfies the following two conditions:

(i) $x \in P, \lambda \geq 0$ imply that $\lambda x \in P$;

(ii) $x \in P,-x \in P$ imply that $x=0$.

Every cone $P \subset E$ induces an ordering in $E$ given by $x \leq y$ if and only if $y-x \in P$.

Definition 2.2 A map $\psi$ is said to be a nonnegative continuous concave functional on a cone $P$ of a real Banach space $E$ if $\psi: P \rightarrow[0, \infty)$ is continuous and

$$
\psi(t x+(1-t) y) \geq t \psi(x)+(1-t) \psi(y)
$$

for all $x, y \in P$ and $t \in[0,1]$.

Similarly, we say the map $\alpha$ is a nonnegative continuous convex functional on a cone $P$ of a real Banach space $E$ if $\alpha: P \rightarrow[0, \infty)$ is continuous and

$$
\alpha(t x+(1-t) y) \leq t \alpha(x)+(1-t) \alpha(y)
$$

for all $x, y \in P$ and $t \in[0,1]$.

Let $\psi$ be a nonnegative continuous concave functional on $P$, and $\alpha$ and $\beta$ be nonnegative continuous convex functionals on $P$.

For nonnegative real numbers $r, a$ and $l$, we define the following convex sets

$$
\begin{aligned}
& P=(\alpha, r ; \beta, l)=\{u \in P: \alpha(u)<r, \beta(u)<l\}, \\
& \bar{P}=(\alpha, r ; \beta, l)=\{u \in P: \alpha(u) \leq r, \beta(u) \leq l\}, \\
& P=(\alpha, r ; \beta, l ; \psi, a)=\{u \in P: \alpha(u)<r, \beta(u)<l, \psi(u)>a\}, \\
& \bar{P}=(\alpha, r ; \beta, l ; \psi, a)=\{u \in P: \alpha(u) \leq r, \beta(u) \leq l, \psi(u) \geq a\} .
\end{aligned}
$$

To prove our main results, we need the following fixed point theorem, which comes from Bai and Ge in [14].

Lemma 2.1 [14] Let P be a cone in a real Banach space E. Assume that constants $r_{1}, b, d, r_{2}$, $l_{1}$ and $l_{2}$ satisfy $0<r_{1}<b<d \leq r_{2}$ and $0<l_{1} \leq l_{2}$. If there exist two nonnegative continuous convex functionals $\alpha$ and $\beta$ on $P$ and a nonnegative continuous concave functional $\psi$ on $P$ such that 
(A1) there exists $M>0$ such that $\|u\| \leq M \max \{\alpha(u), \beta(u)\}$ for all $u \in P$;

(A2) $P(\alpha, r ; \beta, l) \neq \emptyset$ for any $r>0$ and $l>0$;

(A3) $\psi(u) \leq \alpha(u)$ for all $u \in \bar{P}\left(\alpha, r_{2} ; \beta, l_{2}\right)$;

and if $F: \bar{P}\left(\alpha, r_{2} ; \beta, l_{2}\right) \rightarrow\left(\alpha, r_{2} ; \beta, l_{2}\right)$ is completely continuous operator, which satisfies

(B1) $\left\{u \in \bar{P}\left(\alpha, d ; \beta, l_{2} ; \psi, b\right): \psi(u)>b\right\} \neq \emptyset, \psi(F u)>b$ for $u \in \bar{P}\left(\alpha, d ; \beta, l_{2} ; \psi, b\right)$;

(B2) $\alpha(F u)<r_{1}, \beta(F u)<l_{1}$ for $u \in \bar{P}\left(\alpha, r_{1} ; \beta, l_{1}\right)$;

(B3) $\psi(F u)>b$ for $u \in \bar{P}\left(\alpha, r_{2} ; \beta, l_{2} ; \psi, b\right)$ with $\alpha(F u)>d$.

Then $F$ has at least three different fixed points $u_{1}, u_{2}$ and $u_{3}$ in $\bar{P}\left(\alpha, r_{2} ; \beta, l_{2}\right)$ with

$$
u_{1} \in P\left(\alpha, r_{1} ; \beta, l_{1}\right), \quad u_{2} \in\left\{\bar{P}\left(\alpha, r_{2} ; \beta, l_{2} ; \psi, b\right): \psi(u)>b\right\}
$$

and

$$
u_{3} \in \bar{P}\left(\alpha, r_{2} ; \beta, l_{2}\right) \backslash\left(\bar{P}\left(\alpha, r_{2} ; \beta, l_{2} ; \psi, b\right) \cup \bar{P}\left(\alpha, r_{1} ; \beta, l_{1}\right)\right) .
$$

Let the Banach space

$$
\begin{aligned}
E= & C_{\mathrm{ld}}^{1}\left([0, T]_{\mathbb{T}}\right) \\
= & \left\{u\left|[0, T]_{\mathbb{T}} \rightarrow \mathbb{R}\right| u \text { is } \Delta \text {-differentiable on }[0, T]_{\mathbb{T}},\right. \\
& \text { and } \left.u^{\Delta} \text { is ld-continuous on }[0, T]_{\mathbb{T}}\right\}
\end{aligned}
$$

be endowed with the norm

$$
\|u\|=\max \left\{\sup _{t \in[0, T]_{\mathbb{T}}}|u(t)|, \sup _{t \in[0, T]_{\mathbb{T}}}\left|u^{\Delta}(t)\right|\right\} .
$$

Define

$$
P=\left\{u \in E \mid u(t) \geq 0, u^{\Delta}(t) \geq 0 \text {, and } u(t) \text { is concave on }[0, T]_{\mathbb{T}}\right\} .
$$

Clearly, $P$ is a cone.

\section{Existence of triple positive solutions to (1.1) and (1.2)}

Throughout the section, we suppose that the following conditions are satisfied.

(H1) $0, T \in \mathbb{T}, 0<\xi_{1}<\xi_{2}<\cdots<\xi_{n-2}<\rho(T), \xi_{i} \in \mathbb{T}, \alpha_{i}, \beta_{i} \in[0, \infty)$ satisfy

$$
0 \leq \sum_{i=1}^{n-2} \alpha_{i}<1 \text { and } 0 \leq \sum_{i=1}^{n-2} \beta_{i}<1 ;
$$

(H2) $\eta=\min \left\{t \in \mathbb{T}: \frac{T}{2} \leq t<T\right\}$ exists;

(H3) $q(t) \in C_{\mathrm{ld}}\left([0, T]_{\mathbb{T}},[0, \infty)\right)$ with $0<\int_{\eta}^{T} q(t) \Delta t<\infty$;

(H4) $f:(0, T)_{\mathbb{T}} \times[0, \infty) \times \mathbb{R} \rightarrow[0, \infty)$ is continuous;

(H5) $q(t) f(t, 0,0) \not \equiv 0, f(t, 0,0) \geq 0$ on $[0, T]_{\mathbb{T}}$.

Lemma 3.1 If $\sum_{i=1}^{n-2} \alpha_{i} \neq 1$ and $\sum_{i=1}^{n-2} \beta_{i} \neq 1$, then for $h \in C_{\mathrm{ld}}[0, T]_{\mathbb{T}}$,

$$
\begin{aligned}
& \left(\phi_{p}\left(u^{\Delta}(t)\right)\right)^{\Delta}+h(t)=0, \quad t \in(0, T)_{\mathbb{T}}, \\
& u(0)=\sum_{i=1}^{n-2} \alpha_{i} u\left(\xi_{i}\right), \quad \phi_{p}\left(u^{\Delta}(T)\right)=\sum_{i=1}^{n-2} \beta_{i} \phi_{p}\left(u^{\Delta}\left(\xi_{i}\right)\right)
\end{aligned}
$$


has the unique solution

$$
u(t)=\int_{0}^{t} \phi_{q}\left(A+\int_{s}^{T} h(\tau) \Delta \tau\right) \Delta s+B
$$

where

$$
A=\frac{\sum_{i=1}^{n-2} \beta_{i} \int_{\xi_{i}}^{T} h(\tau) \Delta \tau}{1-\sum_{i=1}^{n-2} \beta_{i}}, \quad B=\frac{\sum_{i=1}^{n-2} \alpha_{i}}{1-\sum_{i=1}^{n-2} \alpha_{i}} \int_{0}^{\xi_{i}} \phi_{q}\left(A+\int_{s}^{T} h(\tau) \Delta \tau\right) \Delta s .
$$

Lemma 3.2 The solution of $B V P(3.1)$ and (3.2) satisfies $u \geq 0$, for $t \in[0, T]_{\mathbb{T}}$.

Lemma 3.3 Suppose (H1) holds, if $h \in C_{\mathrm{ld}}[0, T]$ and $h \geq 0$, then the unique solution $u$ of (1.1) and (1.2) satisfies

$$
\inf _{t \in[0, T]_{\mathbb{T}}} u(t) \geq \gamma\|u\|
$$

where

$$
\gamma=\frac{\sum_{i=1}^{n-2} \alpha_{i} \xi_{i}}{T-\sum_{i=1}^{n-2} \alpha_{i}\left(T-\xi_{i}\right)} .
$$

Proof Let

$$
\varphi(s)=\phi_{q}\left(\frac{\sum_{i=1}^{n-2} \beta_{i} \int_{\xi_{i}}^{T} h(\tau) \Delta \tau}{1-\sum_{i=1}^{n-2} \beta_{i}}+\int_{s}^{T} h(\tau) \Delta \tau\right) .
$$

Clearly $u^{\Delta}(t)=\varphi(t) \geq 0$. This implies that

$$
\min _{t \in[0, T]} u(t)=u(0), \quad\|u\|=u(T) .
$$

It is easy to see that $u^{\Delta}\left(t_{2}\right) \leq u^{\Delta}\left(t_{1}\right)$ for any $t_{1}, t_{2} \in[0, T]$ with $t_{1} \leq t_{2}$. Hence $u^{\Delta}(t)$ is a decreasing function on $[0, T]$. This means that the graph of $u(t)$ is concave down on $(0, T)$. For each $i \in\{1,2, \ldots, n-2\}$, we have

$$
\frac{u(T)-u(0)}{T-0} \geq \frac{u(T)-u\left(\xi_{i}\right)}{T-\xi_{i}},
$$

i.e.,

$$
T u\left(\xi_{i}\right)-\xi_{i} u(T) \geq\left(T-\xi_{i}\right) u(0)
$$

so that

$$
T \sum_{i=1}^{n-2} \alpha_{i} u\left(\xi_{i}\right)-\sum_{i=1}^{n-2} \alpha_{i} \xi_{i} u(T) \geq \sum_{i=1}^{n-2} \alpha_{i}\left(T-\xi_{i}\right) u(0) .
$$


With the boundary condition $u(0)=\sum_{i=1}^{n-2} \alpha_{i} u\left(\xi_{i}\right)$, we have

$$
u(0) \geq \frac{\sum_{i=1}^{n-2} \alpha_{i} \xi_{i}}{T-\sum_{i=1}^{n-2} \alpha_{i}\left(T-\xi_{i}\right)} u(T) .
$$

This completes the proof.

Define the operator $F: P \rightarrow E$ by

$$
\begin{aligned}
(F u)(t)= & \int_{0}^{t} \phi_{q}\left(\frac{\sum_{i=1}^{n-2} \beta_{i} \int_{\xi_{i}}^{T} q(\tau) f\left(\tau, u, u^{\Delta}\right) \Delta \tau}{1-\sum_{i=1}^{n-2} \beta_{i}}+\int_{s}^{T} q(\tau) f\left(\tau, u, u^{\Delta}\right) \Delta \tau\right) \Delta s \\
& +\frac{\sum_{i=1}^{n-2} \alpha_{i}}{1-\sum_{i=1}^{n-2} \alpha_{i}} \int_{0}^{\xi_{i}} \phi_{q}\left(\frac{\sum_{i=1}^{n-2} \beta_{i} \int_{\xi_{i}}^{T} q(\tau) f\left(\tau, u, u^{\Delta}\right) \Delta \tau}{1-\sum_{i=1}^{n-2} \beta_{i}}\right. \\
& \left.+\int_{s}^{T} q(\tau) f\left(\tau, u, u^{\Delta}\right) \Delta \tau\right) \Delta s
\end{aligned}
$$

for $t \in[0, T]_{\mathbb{T}}$. By the definition of $F$, the monotonicity of $\phi_{q}(u)$ and the assumptions (H1)(H5), it is easy to see that for each $u \in P, F u \in P$ and $F u(T)$ is the maximum value of $F u(t)$. Moreover, by direct calculation, we get that each fixed point of the operator $F$ in $P$ is a positive solution of the BVP (1.1) and (1.2). It is easy to see that $F: P \rightarrow P$ is completely continuous.

For $u \in P$, we define

$$
\begin{aligned}
& \alpha(u)=\max _{t \in[0, T]_{\mathbb{T}}}|u(t)|=u(T), \quad \beta(u)=\sup _{t \in[0, T]_{\mathbb{T}}}\left|u^{\Delta}(t)\right|=u^{\Delta}(0), \\
& \psi(u)=\min _{t \in[\eta, T]_{\mathbb{T}}} u(t)=u(\eta) .
\end{aligned}
$$

It is easy to see that $\alpha, \beta: P \rightarrow[0, \infty)$ are nonnegative continuous convex functionals with $\|u\|=\max \{\alpha(u), \beta(u)\} ; \psi: P \rightarrow[0, \infty)$ is nonnegative concave functional. We have $\psi(u) \leq$ $\alpha(u)$ for $u \in P$ and the assumptions (A1), (A2) and (A3) in Lemma 2.1 hold.

For notational convenience, we denote $\lambda, L$ and $Q$ by

$$
\begin{aligned}
\lambda= & \int_{0}^{\eta} \phi_{q}\left(\frac{\sum_{i=1}^{n-2} \beta_{i} \int_{\xi_{i}}^{T} q(\tau) \Delta \tau}{1-\sum_{i=1}^{n-2} \beta_{i}}+\int_{\eta}^{T} q(\tau) \Delta \tau\right) \Delta s \\
L= & \int_{0}^{T} \phi_{q}\left(\frac{\sum_{i=1}^{n-2} \beta_{i} \int_{\xi_{i}}^{T} q(\tau) \Delta \tau}{1-\sum_{i=1}^{n-2} \beta_{i}}+\int_{s}^{T} q(\tau) \Delta \tau\right) \Delta s \\
& +\frac{\sum_{i=1}^{n-2} \alpha_{i}}{1-\sum_{i=1}^{n-2} \alpha_{i}} \int_{0}^{\xi_{i}} \phi_{q}\left(\frac{\sum_{i=1}^{n-2} \beta_{i} \int_{\xi_{i}}^{T} q(\tau) \Delta \tau}{1-\sum_{i=1}^{n-2} \beta_{i}}+\int_{s}^{T} q(\tau) \Delta \tau\right) \Delta s, \\
Q= & \phi_{q}\left(\frac{\sum_{i=1}^{n-2} \beta_{i} \int_{\xi_{i}}^{T} q(\tau) \Delta \tau}{1-\sum_{i=1}^{n-2} \beta_{i}}+\int_{0}^{T} q(\tau) \Delta \tau\right) .
\end{aligned}
$$

Theorem 3.1 Assume that (H1)-(H5) hold, and there exists $0<r_{1}<b<2 b \leq r_{2}, 0<l_{1} \leq l_{2}$ such that $\frac{b}{\lambda} \leq \min \left\{r_{2} / L, l_{2} / Q\right\}$. Iff satisfies the following conditions:

(D1) $f(t, w, v) \leq \min \left\{\phi_{p}\left(r_{2} / L\right), \phi_{p}\left(l_{2} / Q\right)\right\}$ for $(t, w, v) \in[0, T]_{\mathbb{T}} \times\left[0, r_{2}\right] \times\left[-l_{2}, l_{2}\right]$;

(D2) $f(t, w, v)>\phi_{p}(b / \lambda)$ for $(t, w, v) \in[\eta, T]_{\mathbb{T}} \times[b, 2 b] \times\left[-l_{2}, l_{2}\right]$; 
(D3) $f(t, w, v)<\min \left\{\phi_{p}\left(r_{1} / L\right), \phi_{p}\left(l_{1} / Q\right)\right\}$ for $(t, w, v) \in[0, T]_{\mathbb{T}} \times\left[0, r_{1}\right] \times\left[-l_{1}, l_{1}\right]$;

then the BVP (1.1) and (1.2) has at least three positive solutions $u_{1}, u_{2}$ and $u_{3}$, which satisfy

$$
\begin{aligned}
& \max _{t \in[0, T]_{\mathbb{T}}}\left\{u_{1}(t)\right\}<r_{1}, \quad \sup _{t \in[0, T]_{\mathbb{T}}}\left|u_{1}^{\Delta}(t)\right|<l_{1} ; \\
& b<\min _{t \in[\eta, T]_{\mathbb{T}}}\left\{u_{2}(t)\right\} \leq \max _{t \in[0, T]_{\mathbb{T}}}\left\{u_{2}(t)\right\} \leq r_{2}, \quad \sup _{t \in[0, T]_{\mathbb{T}}}\left|u_{2}^{\Delta}(t)\right| \leq l_{2} ; \\
& \min _{t \in[\eta, T]_{\mathbb{T}}}\left\{u_{3}(t)\right\}<b, \quad r_{1}<\max _{t \in[0, T]_{\mathbb{T}}}\left\{u_{3}(t)\right\}<2 b, \quad l_{1}<\sup _{t \in[0, T]_{\mathbb{T}}}\left|u_{3}^{\Delta}(t)\right| \leq l_{2} .
\end{aligned}
$$

Proof In order to show that Lemma 2.1 holds, it is sufficient to show that the conditions in Lemma 2.1 are satisfied with respect to operator $F$.

We first prove that if the assumption (D1) is satisfied, then $F: \bar{P}\left(\alpha, r_{2} ; \beta, l_{2}\right) \rightarrow \bar{P}\left(\alpha, r_{2}\right.$; $\left.\beta, l_{2}\right)$. If $u \in \bar{P}\left(\alpha, r_{2} ; \beta, l_{2}\right)$, then

$$
\alpha(u)=\max _{t \in[0, T]_{\mathbb{T}}}|u(t)| \leq r_{2}, \quad \beta(u)=\sup _{t \in[0, T]_{\mathbb{T}}}\left|u^{\Delta}(t)\right| \leq l_{2}
$$

and assumption (D1) implies that

$$
f\left(t, u(t), u^{\Delta}(t)\right) \leq \min \left\{\phi_{p}\left(r_{2} / L\right), \phi_{p}\left(l_{2} / Q\right)\right\}, \quad t \in[0, T]_{\mathbb{T}} .
$$

For $u \in P$, there is $F u \in P$, therefore,

$$
\begin{aligned}
\alpha(F u)= & \max _{t \in[0, T]_{\mathbb{T}}}|(F u)(t)| \\
= & \int_{0}^{T} \phi_{q}\left(\frac{\sum_{i=1}^{n-2} \beta_{i} \int_{\xi_{i}}^{T} q(\tau) f\left(\tau, u, u^{\Delta}\right) \Delta \tau}{1-\sum_{i=1}^{n-2} \beta_{i}}+\int_{s}^{T} q(\tau) f\left(\tau, u, u^{\Delta}\right) \Delta \tau\right) \Delta s \\
& +\frac{\sum_{i=1}^{n-2} \alpha_{i}}{1-\sum_{i=1}^{n-2} \alpha_{i}} \int_{0}^{\xi_{i}} \phi_{q}\left(\frac{\sum_{i=1}^{n-2} \beta_{i} \int_{\xi_{i}}^{T} q(\tau) f\left(\tau, u, u^{\Delta}\right) \Delta \tau}{1-\sum_{i=1}^{n-2} \beta_{i}}\right. \\
& \left.+\int_{s}^{T} q(\tau) f\left(\tau, u, u^{\Delta}\right) \Delta \tau\right) \Delta s \\
\leq & \frac{r_{2}}{L}\left[\int_{0}^{T} \phi_{q}\left(\frac{\sum_{i=1}^{n-2} \beta_{i} \int_{\xi_{i}}^{T} q(\tau) \Delta \tau}{1-\sum_{i=1}^{n-2} \beta_{i}}+\int_{s}^{T} q(\tau) \Delta \tau\right) \Delta s\right. \\
& \left.+\frac{\sum_{i=1}^{n-2} \alpha_{i}}{1-\sum_{i=1}^{n-2} \alpha_{i}} \int_{0}^{\xi_{i}} \phi_{q}\left(\frac{\sum_{i=1}^{n-2} \beta_{i} \int_{\xi_{i}}^{T} q(\tau) \Delta \tau}{1-\sum_{i=1}^{n-2} \beta_{i}}+\int_{s}^{T} q(\tau) \Delta \tau\right) \Delta s\right]=r_{2}
\end{aligned}
$$

and

$$
\begin{aligned}
\beta(F u) & =\sup _{t \in[0, T]_{\mathbb{T}}}\left|(F u)^{\Delta}(t)\right| \\
& =\phi_{q}\left(\frac{\sum_{i=1}^{n-2} \beta_{i} \int_{\xi_{i}}^{T} q(\tau) f\left(\tau, u, u^{\Delta}\right) \Delta \tau}{1-\sum_{i=1}^{n-2} \beta_{i}}+\int_{0}^{T} q(\tau) f\left(\tau, u, u^{\Delta}\right) \Delta \tau\right) \\
& \leq \frac{l_{2}}{Q} \phi_{q}\left(\frac{\sum_{i=1}^{n-2} \beta_{i} \int_{\xi_{i}}^{T} q(\tau) \Delta \tau}{1-\sum_{i=1}^{n-2} \beta_{i}}+\int_{0}^{T} q(\tau) \Delta \tau\right)=l_{2} .
\end{aligned}
$$

Therefore, $F: \bar{P}\left(\alpha, r_{2} ; \beta, l_{2}\right) \rightarrow \bar{P}\left(\alpha, r_{2} ; \beta, l_{2}\right)$. 
Similarly, if $u \in \bar{P}\left(\alpha, r_{1} ; \beta, l_{1}\right)$, then the assumption (D3) implies that

$$
f\left(t, u(t), u^{\Delta}(t)\right)<\min \left\{\phi_{p}\left(r_{1} / L\right), \phi_{p}\left(l_{1} / Q\right)\right\} \quad \text { for } t \in[0, T]_{\mathbb{T}} .
$$

We can get that $F: \bar{P}\left(\alpha, r_{1} ; \beta, l_{1}\right) \rightarrow P\left(\alpha, r_{1} ; \beta, l_{1}\right)$.

So condition (B2) of Lemma 2.1 is satisfied.

To prove condition (B1) of Lemma 2.1 holds. We choose $u(t)=2 b$ for $t \in[0, T]_{\mathbb{T}}$. It is obvious that

$$
u(t)=2 b \in \bar{P}\left(\alpha, 2 b ; \beta, l_{2} ; \psi, b\right) \quad \text { and } \quad \psi(u)=2 b>b
$$

and, consequently,

$$
\left\{u \in \bar{P}\left(\alpha, 2 b ; \beta, l_{2} ; \psi, b\right): \psi(u)>b\right\} \neq \emptyset .
$$

So, for $u \in \bar{P}\left(\alpha, 2 b ; \beta, l_{2} ; \psi, b\right)$, there are $b \leq u(t) \leq 2 b$ and $\left|u^{\Delta}(t)\right| \leq l_{2}$ for $t \in[\eta, T]_{\mathbb{T}}$.

Thus, from the assumption (D2), we have

$$
f\left(t, u(t), u^{\Delta}(t)\right)>\phi_{p}(b / \lambda) \quad \text { for } t \in[\eta, T]_{\mathbb{T}} .
$$

From the definition of the functional $\psi$, we see that

$$
\begin{aligned}
\psi(F u)= & \min _{t \in[\eta, T]_{\mathbb{T}}} F u(t)=F u(\eta) \\
= & \int_{0}^{\eta} \phi_{q}\left(\frac{\sum_{i=1}^{n-2} \beta_{i} \int_{\xi_{i}}^{T} q(\tau) f\left(\tau, u, u^{\Delta}\right) \Delta \tau}{1-\sum_{i=1}^{n-2} \beta_{i}}+\int_{s}^{T} q(\tau) f\left(\tau, u, u^{\Delta}\right) \Delta \tau\right) \Delta s \\
& +\frac{\sum_{i=1}^{n-2} \alpha_{i}}{1-\sum_{i=1}^{n-2} \alpha_{i}} \int_{0}^{\xi_{i}} \phi_{q}\left(\frac{\sum_{i=1}^{n-2} \beta_{i} \int_{\xi_{i}}^{T} q(\tau) f\left(\tau, u, u^{\Delta}\right) \Delta \tau}{1-\sum_{i=1}^{n-2} \beta_{i}}\right. \\
& \left.+\int_{s}^{T} q(\tau) f\left(\tau, u, u^{\Delta}\right) \Delta \tau\right) \Delta s \\
\geq & \int_{0}^{\eta} \phi_{q}\left(\frac{\sum_{i=1}^{n-2} \beta_{i} \int_{\xi_{i}}^{T} q(\tau) f\left(\tau, u, u^{\Delta}\right) \Delta \tau}{1-\sum_{i=1}^{n-2} \beta_{i}}+\int_{\eta}^{T} q(\tau) f\left(\tau, u, u^{\Delta}\right) \Delta \tau\right) \Delta s \\
> & \int_{0}^{\eta} \phi_{q}\left(\frac{\sum_{i=1}^{n-2} \beta_{i} \int_{\xi_{i}}^{T} q(\tau) \phi_{p}(b / \lambda) \Delta \tau}{1-\sum_{i=1}^{n-2} \beta_{i}}+\int_{\eta}^{T} q(\tau) \phi_{p}(b / \lambda) \Delta \tau\right) \Delta s \\
= & \frac{b}{\lambda} \int_{0}^{\eta} \phi_{q}\left(\frac{\sum_{i=1}^{n-2} \beta_{i} \int_{\xi_{i}}^{T} q(\tau) \Delta \tau}{1-\sum_{i=1}^{n-2} \beta_{i}}+\int_{\eta}^{T} q(\tau) \Delta \tau\right) \Delta s=b .
\end{aligned}
$$

So, we get $\psi(F u)>b$ for $u \in \bar{P}\left(\alpha, 2 b ; \beta, l_{2} ; \psi, b\right)$, and condition (B1) of Lemma 2.1 holds.

Finally, we prove that condition (B3) of Lemma 2.1 holds.

If $u \in \bar{P}\left(\alpha, r_{2} ; \beta, l_{2} ; \psi, b\right)$ and $\alpha(F u)>2 b$, we have

$$
\psi(F u)=\min _{t \in[\eta, T]_{\mathbb{T}}} F u(t)=F u(\eta) \geq \frac{\eta}{T} \max _{t \in[0, T]_{\mathbb{T}}} F u(t) \geq \frac{1}{2} \alpha(F u)>b .
$$


Hence, condition (B3) of Lemma 2.1 is satisfied. Then using Lemma 2.1 and the assumption that $f(t, 0,0) \not \equiv 0$ on $[0, T]_{\mathbb{T}}$, we find that there exist at least three positive solutions of (1.1) and (1.2) such that

$$
u_{1} \in P\left(\alpha, r_{1} ; \beta, l_{1}\right), \quad u_{2} \in\left\{P\left(\alpha, r_{2} ; \beta, l_{2} ; \psi, b\right) \mid \psi(u)>b\right\}
$$

and

$$
u_{3} \in \bar{P}\left(\alpha, r_{2} ; \beta, l_{2}\right) \backslash\left(\bar{P}\left(\alpha, r_{2} ; \beta, l_{2} ; \psi, b\right) \cup \bar{P}\left(\alpha, r_{1} ; \beta, l_{1}\right)\right)
$$

Otherwise, as $u_{3}$ satisfies $\alpha\left(u_{3}\right) \leq 2 \psi\left(u_{3}\right)$, we have $\max _{t \in[0, T]_{\mathbb{T}}} u_{3}(t)<2 b$.

Example 3.1 Let $\mathbb{T}=\left\{1-\left(\frac{1}{2}\right)^{\mathbb{N}_{0}}\right\} \cup[1,2], \mathbb{N}_{0}$ denotes the set of all nonnegative integers. Take $\alpha_{1}=\frac{1}{2}, \alpha_{2}=\frac{1}{6}, \beta_{1}=\frac{1}{3}, \beta_{2}=\frac{1}{6}, \xi_{1}=\frac{1}{4}, \xi_{2}=\frac{3}{4}, T=2, p=q=2$ and $q(t) \equiv 1, t \in[0, T]_{\mathbb{T}}$. Consider the following BVP

$$
\begin{array}{ll}
\left(u^{\Delta}(t)\right)^{\Delta}+f\left(t, u(t), u^{\Delta}(t)\right)=0, & t \in[0,2]_{\mathbb{T}}, \\
u(0)=\frac{1}{2} u\left(\frac{1}{4}\right)+\frac{1}{6} u\left(\frac{3}{4}\right), & u^{\Delta}(2)=\frac{1}{3}\left(u^{\Delta}\left(\frac{1}{4}\right)\right)+\frac{1}{6}\left(u^{\Delta}\left(\frac{3}{4}\right)\right),
\end{array}
$$

where

$$
f(t, w, v)= \begin{cases}\frac{t}{1,000}+\frac{w^{3}}{3}+\left(\frac{v}{100}\right)^{3}, & w \leq 3 \\ \frac{t}{1,000}+9+\left(\frac{v}{100}\right)^{3}, & w>3\end{cases}
$$

Clearly, the assumptions (H1)-(H5) hold, and $f(t, 0,0) \not \equiv 0$ on $[0,2]_{\mathbb{T}}$.

We choose $r_{1}=1 / 2, r_{2}=140, b=2$ and $l_{1}=1 / 4, l_{2}=80$. So $0<r_{1}<b<2 b<r_{2}$ and $0<l_{1}<l_{2}$. By calculating, we obtain

$$
\begin{aligned}
& \lambda=\int_{0}^{\eta} \phi_{q}\left(\frac{\sum_{i=1}^{n-2} \beta_{i} \int_{\xi_{i}}^{T} q(\tau) \Delta \tau}{1-\sum_{i=1}^{n-2} \beta_{i}}+\int_{\eta}^{T} q(\tau) \Delta \tau\right) \Delta s=\frac{31}{12}, \\
& Q=\phi_{q}\left(\frac{\sum_{i=1}^{n-2} \beta_{i} \int_{\xi_{i}}^{T} q(\tau) \Delta \tau}{1-\sum_{i=1}^{n-2} \beta_{i}}+\int_{0}^{T} q(\tau) \Delta \tau\right)=\frac{43}{12}
\end{aligned}
$$

and

$$
\begin{aligned}
L= & \int_{0}^{T} \phi_{q}\left(\frac{\sum_{i=1}^{n-2} \beta_{i} \int_{\xi_{i}}^{T} q(\tau) \Delta \tau}{1-\sum_{i=1}^{n-2} \beta_{i}}+\int_{s}^{T} q(\tau) \Delta \tau\right) \Delta s \\
& +\frac{\sum_{i=1}^{n-2} \alpha_{i}}{1-\sum_{i=1}^{n-2} \alpha_{i}} \int_{0}^{\xi_{i}} \phi_{q}\left(\frac{\sum_{i=1}^{n-2} \beta_{i} \int_{\xi_{i}}^{T} q(\tau) \Delta \tau}{1-\sum_{i=1}^{n-2} \beta_{i}}+\int_{s}^{T} q(\tau) \Delta \tau\right) \Delta s<\tilde{L} \\
= & \int_{0}^{T} \phi_{q}\left(\frac{\sum_{i=1}^{n-2} \beta_{i} \int_{\xi_{i}}^{T} q(\tau) \Delta \tau}{1-\sum_{i=1}^{n-2} \beta_{i}}+\int_{0}^{T} q(\tau) \Delta \tau\right) \Delta s \\
& +\frac{\sum_{i=1}^{n-2} \alpha_{i}}{1-\sum_{i=1}^{n-2} \alpha_{i}} \int_{0}^{\xi_{i}} \phi_{q}\left(\frac{\sum_{i=1}^{n-2} \beta_{i} \int_{\xi_{i}}^{T} q(\tau) \Delta \tau}{1-\sum_{i=1}^{n-2} \beta_{i}}+\int_{0}^{T} q(\tau) \Delta \tau\right) \Delta s=\frac{473}{48} .
\end{aligned}
$$


As a result, $f(t, w, v)$ satisfies

$$
\begin{aligned}
& f(t, w, v) \leq \min \left\{\phi_{p}\left(\frac{r_{2}}{\tilde{L}}\right), \phi_{p}\left(\frac{l_{2}}{Q}\right)\right\} \approx 14.2071<\min \left\{\phi_{p}\left(\frac{r_{2}}{L}\right), \phi_{p}\left(\frac{l_{2}}{Q}\right)\right\} \\
& \text { for } 0 \leq t \leq 2,0 \leq w \leq 140,|v| \leq 80 \\
& f(t, w, v)>\phi_{p}\left(\frac{b}{\lambda}\right) \approx 0.7742 \text { for } 1 \leq t \leq 2,2 \leq w \leq 4,|v| \leq 80 ; \\
& f(t, w, v)<\min \left\{\phi_{p}\left(\frac{r_{1}}{\tilde{L}}\right), \phi_{p}\left(\frac{l_{1}}{Q}\right)\right\} \approx 0.0507<\min \left\{\phi_{p}\left(\frac{r_{1}}{L}\right), \phi_{p}\left(\frac{l_{1}}{Q}\right)\right\} \\
& \quad \text { for } 0 \leq t \leq 2,0 \leq w \leq \frac{1}{2},|v| \leq \frac{1}{4} .
\end{aligned}
$$

Hence, by Theorem 3.1, we have that the BVP (3.4) and (3.5) has at least three positive solutions $u_{1}, u_{2}$ and $u_{3}$ such that

$$
\begin{aligned}
& \max _{t \in[0,2]_{\mathbb{T}}}\left\{u_{1}(t)\right\}<\frac{1}{2}, \quad \sup _{t \in[0, T]_{\mathbb{T}}}\left|u_{1}^{\Delta}(t)\right|<\frac{1}{4} ; \\
& 2<\min _{t \in[1,2]_{\mathbb{T}}}\left\{u_{2}(t)\right\} \leq \max _{t \in[0,2]_{\mathbb{T}}}\left\{u_{2}(t)\right\} \leq 140, \quad \sup _{t \in[0,2]_{\mathbb{T}}}\left|u_{2}^{\Delta}(t)\right| \leq 80 ; \\
& \min _{t \in[1,2]_{\mathbb{T}}}\left\{u_{3}(t)\right\}<2, \quad \frac{1}{2}<\max _{t \in[0,2]_{\mathbb{T}}}\left\{u_{3}(t)\right\}<4, \quad \frac{1}{4}<\sup _{t \in[0,2]_{\mathbb{T}}}\left|u_{3}^{\Delta}(t)\right| \leq 80 .
\end{aligned}
$$

\section{Existence of triple positive solutions to (1.3) and (1.4)}

The following conditions will be used in this section:

(H1) $0, T \in \mathbb{T}, 0<\xi_{1}<\xi_{2}<\cdots<\xi_{m-2}<\rho(T), \xi_{i} \in \mathbb{T}, a_{i}, b_{i} \in[0, \infty)$ satisfy

$$
0 \leq \sum_{i=1}^{m-2} a_{i}<1 \text { and } 0 \leq \sum_{i=1}^{m-2} b_{i}<1 \text {; }
$$

(H2) $\eta=\min \{t \in \mathbb{T}: T / 2 \leq t<T\}$ exists;

(H3) $a(t) \in C_{\mathrm{ld}}\left([0, T]_{\mathbb{T}},[0, \infty)\right)$ with $0<\int_{\eta}^{T} a(t) \nabla t<\infty$;

(H4) $f:(0, T)_{\mathbb{T}} \times[0, \infty) \times \mathbb{R} \rightarrow[0, \infty)$ is continuous;

(H5) $a(t) f(t, 0,0) \not \equiv 0, f(t, 0,0) \geq 0$ on $[0, T]_{\mathbb{T}}$.

Let the Banach space

$$
\begin{aligned}
E= & C_{\mathrm{ld}}^{1}\left([0, T]_{\mathbb{T}}\right) \\
= & \left\{u\left|[0, T]_{\mathbb{T}} \rightarrow \mathbb{R}\right| u \text { is } \Delta \text {-differentiable on }[0, T]_{\mathbb{T}},\right. \\
& \text { and } \left.u^{\Delta} \text { is ld-continuous on }[0, T]_{\mathbb{T}}\right\}
\end{aligned}
$$

be endowed with the norm

$$
\|u\|=\max \left\{\sup _{t \in[0, T]_{\mathbb{T}}}|u(t)|, \sup _{t \in[0, T]_{\mathbb{T}}}\left|u^{\Delta}(t)\right|\right\} .
$$

Define

$$
K=\left\{u \in E \mid u(t) \geq 0, u^{\Delta}(t) \leq 0, \text { and } u(t) \text { is concave on }[0, T]_{\mathbb{T}}\right\}
$$

Clearly, $K$ is a cone. 
We note that $u(t)$ is a solution of (1.3) and (1.4) if and only if

$$
\begin{aligned}
u(t)= & \int_{t}^{T} \phi_{q}\left(\frac{\sum_{i=1}^{m-2} a_{i} \int_{0}^{\xi_{i}} a(r) f\left(r, u, u^{\Delta}\right) \nabla r}{1-\sum_{i=1}^{m-2} a_{i}}+\int_{0}^{s} a(r) f\left(r, u, u^{\Delta}\right) \nabla r\right) \Delta s \\
& +\frac{\sum_{i=1}^{m-2} b_{i}}{1-\sum_{i=1}^{m-2} b_{i}} \int_{\xi_{i}}^{T} \phi_{q}\left(\frac{\sum_{i=1}^{m-2} a_{i} \int_{0}^{\xi_{i}} a(r) f\left(r, u, u^{\Delta}\right) \nabla r}{1-\sum_{i=1}^{m-2} a_{i}}\right. \\
& \left.+\int_{0}^{s} a(r) f\left(r, u, u^{\Delta}\right) \nabla r\right) \Delta s .
\end{aligned}
$$

Define the operator $A: K \rightarrow E$ by

$$
\begin{aligned}
(A u)(t)= & \int_{t}^{T} \phi_{q}\left(\frac{\sum_{i=1}^{m-2} a_{i} \int_{0}^{\xi_{i}} a(r) f\left(r, u, u^{\Delta}\right) \nabla r}{1-\sum_{i=1}^{m-2} a_{i}}+\int_{0}^{s} a(r) f\left(r, u, u^{\Delta}\right) \nabla r\right) \Delta s \\
& +\frac{\sum_{i=1}^{m-2} b_{i}}{1-\sum_{i=1}^{m-2} b_{i}} \int_{\xi_{i}}^{T} \phi_{q}\left(\frac{\sum_{i=1}^{m-2} a_{i} \int_{0}^{\xi_{i}} a(r) f\left(r, u, u^{\Delta}\right) \nabla r}{1-\sum_{i=1}^{m-2} a_{i}}\right. \\
& \left.+\int_{0}^{s} a(r) f\left(r, u, u^{\Delta}\right) \nabla r\right) \Delta s
\end{aligned}
$$

for $0 \leq t \leq T$. By the definition of $A$, the monotonicity of $\phi_{q}(u)$ and assumptions (H1)(H5), it is easy to see that for each $u \in K, A u \in K$ and $A u(0)$ is the maximum value of $A u(t)$. Moreover, by direct calculation, we get that each fixed point of the operator $A$ in $K$ is a positive solution of (1.3), (1.4). It is easy to see that $A: K \rightarrow K$ is completely continuous.

For $u \in K$, we define

$$
\begin{aligned}
& \alpha(u)=\max _{t \in[0, T]_{\mathbb{T}}}|u(t)|=u(0), \quad \beta(u)=\sup _{t \in[0, T]_{\mathbb{T}}}\left|u^{\Delta}(t)\right|=u^{\Delta}(T), \\
& \psi(u)=\min _{t \in[\eta, T]_{\mathbb{T}}} u(t)=u(T) .
\end{aligned}
$$

It is easy to see that $\alpha, \beta: K \rightarrow[0, \infty)$ are nonnegative continuous convex functionals with $\|u\|=\max \{\alpha(u), \beta(u)\} ; \psi: K \rightarrow[0, \infty)$ is nonnegative concave functional. We have $\psi(u) \leq \alpha(u)$ for $u \in K$ and assumptions (A1), (A2) and (A3) in Lemma 2.1 hold.

For notational convenience, we denote $M, N$ and $Q$ by

$$
\begin{aligned}
M= & \frac{\sum_{i=1}^{m-2} b_{i}}{1-\sum_{i=1}^{m-2} b_{i}} \int_{\xi_{i}}^{T} \phi_{q}\left(\frac{\sum_{i=1}^{m-2} a_{i} \int_{0}^{\xi_{i}} a(r) \nabla r}{1-\sum_{i=1}^{m-2} a_{i}}+\int_{0}^{\eta} a(r) \nabla r\right) \Delta s, \\
N= & \int_{0}^{T} \phi_{q}\left(\frac{\sum_{i=1}^{m-2} a_{i} \int_{0}^{\xi_{i}} a(r) \nabla r}{1-\sum_{i=1}^{m-2} a_{i}}+\int_{0}^{s} a(r) \nabla r\right) \Delta s \\
& +\frac{\sum_{i=1}^{m-2} b_{i}}{1-\sum_{i=1}^{m-2} b_{i}} \int_{\xi_{i}}^{T} \phi_{q}\left(\frac{\sum_{i=1}^{m-2} a_{i} \int_{0}^{\xi_{i}} a(r) \nabla r}{1-\sum_{i=1}^{m-2} a_{i}}+\int_{0}^{s} a(r) \nabla r\right) \Delta s, \\
Q= & \phi_{q}\left(\frac{\sum_{i=1}^{m-2} a_{i} \int_{0}^{\xi_{i}} a(r) \nabla r}{1-\sum_{i=1}^{m-2} a_{i}}+\int_{0}^{T} a(r) \nabla r\right) .
\end{aligned}
$$

Theorem 4.1 Assume that (H1)-(H5) hold, and there exists $0<r_{1}<b<2 b \leq r_{2}, 0<l_{1} \leq l_{2}$ such that $b / M \leq \min \left\{r_{2} / N, l_{2} / Q\right\}$. Iff satisfies the following conditions:

(D1) $f(t, w, v) \leq \min \left\{\phi_{p}\left(r_{2} / N\right), \phi_{p}\left(l_{2} / Q\right)\right\}$ for $(t, w, v) \in[0, T]_{\mathbb{T}} \times\left[0, r_{2}\right] \times\left[-l_{2}, l_{2}\right]$; 
(D2) $f(t, w, v)>\phi_{p}(b / M)$ for $(t, w, v) \in[\eta, T]_{\mathbb{T}} \times[b, 2 b] \times\left[-l_{2}, l_{2}\right]$;

(D3) $f(t, w, v)<\min \left\{\phi_{p}\left(r_{1} / N\right), \phi_{p}\left(l_{1} / Q\right)\right\}$ for $(t, w, v) \in[0, T]_{\mathbb{T}} \times\left[0, r_{1}\right] \times\left[-l_{1}, l_{1}\right]$,

then the BVP (1.3) and (1.4) has at least three positive solutions $u_{1}, u_{2}$ and $u_{3}$, which satisfy

$$
\begin{aligned}
& \max _{t \in[0, T]_{\mathbb{T}}}\left\{u_{1}(t)\right\}<r_{1}, \quad \sup _{t \in[0, T]_{\mathbb{T}}}\left|u_{1}^{\Delta}(t)\right|<l_{1} ; \\
& b<\min _{t \in[\eta, T]_{\mathbb{T}}}\left\{u_{2}(t)\right\} \leq \max _{t \in[0, T]_{\mathbb{T}}}\left\{u_{2}(t)\right\} \leq r_{2}, \quad \sup _{t \in[0, T]_{\mathbb{T}}}\left|u_{2}^{\Delta}(t)\right| \leq l_{2} ; \\
& \min _{t \in[\eta, T]_{\mathbb{T}}}\left\{u_{3}(t)\right\}<b, \quad \max _{t \in[0, T]_{\mathbb{T}}}\left\{u_{3}(t)\right\}<2 b, \quad \sup _{t \in[0, T]_{\mathbb{T}}}\left|u_{3}^{\Delta}(t)\right| \leq l_{2} .
\end{aligned}
$$

Proof In order to show that Lemma 2.1 holds, it is sufficient to show that the conditions in Lemma 2.1 are satisfied with respect to the operator $A$.

We first prove that if the assumption (D1) is satisfied, then $A: \bar{P}\left(\alpha, r_{2} ; \beta, l_{2}\right) \rightarrow \bar{P}\left(\alpha, r_{2}\right.$; $\left.\beta, l_{2}\right)$. If $u \in \bar{P}\left(\alpha, r_{2} ; \beta, l_{2}\right)$, then

$$
\alpha(u)=\max _{t \in[0, T]_{\mathbb{T}}}|u(t)| \leq r_{2}, \quad \beta(u)=\sup _{t \in[0, T]_{\mathbb{T}}}\left|u^{\Delta}(t)\right| \leq l_{2}
$$

and assumption (D1) implies that

$$
f\left(t, u(t), u^{\Delta}(t)\right) \leq \min \left\{\phi_{p}\left(r_{2} / N\right), \phi_{p}\left(l_{2} / Q\right)\right\}, \quad t \in[0, T]_{\mathbb{T}} .
$$

For $u \in K$, there is $A u \in K$, therefore,

$$
\begin{aligned}
\alpha(A u)= & \max _{t \in[0, T]_{\mathbb{T}}}|(A u)(t)| \\
= & \int_{0}^{T} \phi_{q}\left(\frac{\sum_{i=1}^{m-2} a_{i} \int_{0}^{\xi_{i}} a(r) f\left(r, u, u^{\Delta}\right) \nabla r}{1-\sum_{i=1}^{m-2} a_{i}}+\int_{0}^{s} a(r) f\left(r, u, u^{\Delta}\right) \nabla r\right) \Delta s \\
& +\frac{\sum_{i=1}^{m-2} b_{i}}{1-\sum_{i=1}^{m-2} b_{i}} \int_{\xi_{i}}^{T} \phi_{q}\left(\frac{\sum_{i=1}^{m-2} a_{i} \int_{0}^{\xi_{i}} a(r) f\left(r, u, u^{\Delta}\right) \nabla r}{1-\sum_{i=1}^{m-2} a_{i}}\right. \\
& \left.+\int_{0}^{s} a(r) f\left(r, u, u^{\Delta}\right) \nabla r\right) \Delta s \\
< & \frac{r_{2}}{N}\left[\int_{0}^{T} \phi_{q}\left(\frac{\sum_{i=1}^{m-2} a_{i} \int_{0}^{\xi_{i}} a(r) \nabla r}{1-\sum_{i=1}^{m-2} a_{i}}+\int_{0}^{s} a(r) \nabla r\right) \Delta s\right. \\
& \left.+\frac{\sum_{i=1}^{m-2} b_{i}}{1-\sum_{i=1}^{m-2} b_{i}} \int_{\xi_{i}}^{T} \phi_{q}\left(\frac{\sum_{i=1}^{m-2} a_{i} \int_{0}^{\xi_{i}} a(r) \nabla r}{1-\sum_{i=1}^{m-2} a_{i}}+\int_{0}^{s} a(r) \nabla r\right) \Delta s\right]=r_{2}
\end{aligned}
$$

and

$$
\begin{aligned}
\beta(A u) & =\sup _{t \in[0, T]_{\mathbb{T}}}\left|(A u)^{\Delta}(t)\right| \\
& =\phi_{q}\left(\int_{0}^{T} a(r) f\left(r, u, u^{\Delta}\right) \nabla r+\frac{\sum_{i=1}^{m-2} a_{i}}{1-\sum_{i=1}^{m-2} a_{i}} \int_{0}^{\xi_{i}} a(r) f\left(r, u, u^{\Delta}\right) \nabla r\right) \\
& \leq \frac{l_{2}}{Q}\left(\phi_{q}\left(\int_{0}^{T} a(r) \nabla r+\frac{\sum_{i=1}^{m-2} a_{i}}{1-\sum_{i=1}^{m-2} a_{i}} \int_{0}^{\xi_{i}} a(r) \nabla r\right)\right)=l_{2} .
\end{aligned}
$$

Therefore, $A: \bar{P}\left(\alpha, r_{2} ; \beta, l_{2}\right) \rightarrow \bar{P}\left(\alpha, r_{2} ; \beta, l_{2}\right)$. 
Similarly if $u \in \bar{P}\left(\alpha, r_{1} ; \beta, l_{1}\right)$, then the assumption (D3) implies that

$$
f\left(t, u(t), u^{\Delta}(t)\right)<\min \left\{\phi_{p}\left(r_{1} / N\right), \phi_{p}\left(l_{1} / Q\right)\right\} \quad \text { for } t \in[0, T]_{\mathbb{T}} .
$$

We can get that $A: \bar{P}\left(\alpha, r_{1} ; \beta, l_{1}\right) \rightarrow P\left(\alpha, r_{1} ; \beta, l_{1}\right)$.

So condition (B2) of Lemma 2.1 is satisfied.

To prove condition (B1) of Lemma 2.1 holds. We choose $u(t)=2 b$ for $t \in[0, T]_{\mathbb{T}}$. It is obvious that

$$
u(t)=2 b \in \bar{P}\left(\alpha, 2 b ; \beta, l_{2} ; \psi, b\right) \text { and } \quad \psi(u)=2 b>b,
$$

and, consequently,

$$
\left\{u \in \bar{P}\left(\alpha, 2 b ; \beta, l_{2} ; \psi, b\right): \psi(u)>b\right\} \neq \emptyset .
$$

So, for $u \in \bar{P}\left(\alpha, 2 b ; \beta, l_{2} ; \psi, b\right)$, there are $b \leq u(t) \leq 2 b$ and $\left|u^{\Delta}(t)\right| \leq l_{2}$ for $t \in[\eta, T]_{\mathbb{T}}$.

Thus, from the assumption (D2), we have

$$
f\left(t, u(t), u^{\Delta}(t)\right)>\phi_{p}(b / M) \quad \text { for } t \in[\eta, T]_{\mathbb{T}} .
$$

From the definition of the functional $\psi$, we see that

$$
\begin{aligned}
\psi(A u)= & \min _{t \in[\eta, T]_{\mathbb{T}}} A u(t) \\
= & \frac{\sum_{i=1}^{m-2} b_{i}}{1-\sum_{i=1}^{m-2} b_{i}} \int_{\xi_{i}}^{T} \phi_{q}\left(\frac{\sum_{i=1}^{m-2} a_{i} \int_{0}^{\xi_{i}} a(r) f\left(r, u, u^{\Delta}\right) \nabla r}{1-\sum_{i=1}^{m-2} a_{i}}\right. \\
& \left.+\int_{0}^{s} a(r) f\left(r, u, u^{\Delta}\right) \nabla r\right) \Delta s \\
\geq & \frac{\sum_{i=1}^{m-2} b_{i}}{1-\sum_{i=1}^{m-2} b_{i}} \int_{\xi_{i}}^{T} \phi_{q}\left(\frac{\sum_{i=1}^{m-2} a_{i} \int_{0}^{\xi_{i}} a(r) f\left(r, u, u^{\Delta}\right) \nabla r}{1-\sum_{i=1}^{m-2} a_{i}}\right. \\
& \left.+\int_{0}^{\eta} a(r) f\left(r, u, u^{\Delta}\right) \nabla r\right) \Delta s \\
> & \frac{\sum_{i=1}^{m-2} b_{i}}{1-\sum_{i=1}^{m-2} b_{i}} \int_{\xi_{i}}^{T} \phi_{q}\left(\frac{\sum_{i=1}^{m-2} a_{i} \int_{0}^{\xi_{i}} a(r) \phi_{p}(b / M) \nabla r}{1-\sum_{i=1}^{m-2} a_{i}}+\int_{0}^{\eta} a(r) \phi_{p}(b / M) \nabla r\right) \Delta s \\
= & \frac{b}{M}\left(\frac{\sum_{i=1}^{m-2} b_{i}}{1-\sum_{i=1}^{m-2} b_{i}} \int_{\xi_{i}}^{T} \phi_{q}\left(\frac{\sum_{i=1}^{m-2} a_{i} \int_{0}^{\xi_{i}} a(r) \nabla r}{1-\sum_{i=1}^{m-2} a_{i}}+\int_{0}^{\eta} a(r) \nabla r\right) \Delta s\right)=b .
\end{aligned}
$$

So, we get $\psi(A u)>b$ for $u \in \bar{P}\left(\alpha, 2 b ; \beta, l_{2} ; \psi, b\right)$, and condition (B1) of Lemma 2.1 holds.

Finally, we prove that condition (B3) of Lemma 2.1 holds. If $u \in \bar{P}\left(\alpha, r_{2} ; \beta, l_{2} ; \psi, b\right)$ and $\alpha(A u)>2 b$, we have

$$
\psi(A u)=\min _{t \in[\eta, T]_{\mathbb{T}}} A u(t)=A u(T) \geq \max _{t \in[0, T]_{\mathbb{T}}} A u(t) \geq \alpha(A u)>2 b>b .
$$

Hence, condition (B3) of Lemma 2.1 is satisfied. Then using Lemma 2.1 and the assumption that $f(t, 0,0) \not \equiv 0$ on $[0, T]_{\mathbb{T}}$, we find that there exist at least three positive solutions of (1.3) 
and (1.4) such that

$$
u_{1} \in P\left(\alpha, r_{1} ; \beta, l_{1}\right), \quad u_{2} \in\left\{P\left(\alpha, r_{2} ; \beta, l_{2} ; \psi, b\right) \mid \psi(u)>b\right\}
$$

and

$u_{3} \in \bar{P}\left(\alpha, r_{2} ; \beta, l_{2}\right) \backslash\left(\bar{P}\left(\alpha, r_{2} ; \beta, l_{2} ; \psi, b\right) \cup \bar{P}\left(\alpha, r_{1} ; \beta, l_{1}\right)\right)$.

Otherwise, as $u_{3}$ satisfies $\alpha\left(u_{3}\right) \leq 2 \psi\left(u_{3}\right)$, we have $\max _{t \in[0, T]_{\mathbb{T}}} u_{3}(t)<2 b$.

Example 4.1 Let $\mathbb{T}=\left\{1-(1 / 2)^{\mathbb{N}_{0}}\right\} \cup[1,2], \mathbb{N}_{0}$ denotes the set of all nonnegative integers. Take $a_{1}=1 / 2, a_{2}=1 / 6, b_{1}=1 / 3, b_{2}=1 / 6, \xi_{1}=1 / 4, \xi_{2}=3 / 4, T=2, p=q=2$ and $a(t) \equiv 1$, $t \in[0, T]_{\mathbb{T}}$. Consider the following BVP

$$
\begin{aligned}
& \left(u^{\Delta}(t)\right)^{\nabla}+f\left(t, u(t), u^{\Delta}(t)\right)=0, \quad t \in[0,2]_{\mathbb{T}}, \\
& u^{\Delta}(0)=\frac{1}{2}\left(u^{\Delta}\left(\frac{1}{4}\right)\right)+\frac{1}{6}\left(u^{\Delta}\left(\frac{3}{4}\right)\right), \quad u(2)=\frac{1}{3} u\left(\frac{1}{4}\right)+\frac{1}{6} u\left(\frac{3}{4}\right),
\end{aligned}
$$

where

$$
f(t, w, v)= \begin{cases}\frac{t^{2}}{1,000}+\frac{4 w^{3}}{9}+\left(\frac{v}{100,000}\right)^{3}, & w \leq 3, \\ \frac{t^{2}}{1,000}+12+\left(\frac{v}{100,000}\right)^{3}, & w>3 .\end{cases}
$$

Clearly, the assumptions (H1)-(H5) hold, and $f(t, 0,0) \not \equiv 0$ on $[0,2]_{\mathbb{T}}$.

We choose $r_{1}=1 / 8, r_{2}=140, b=2$ and $l_{1}=1 / 4, l_{2}=80$. So $0<r_{1}<b<2 b<r_{2}$ and $0<l_{1}<l_{2}$. By calculating, we obtain

$$
\begin{aligned}
& M=\frac{\sum_{i=1}^{m-2} b_{i}}{1-\sum_{i=1}^{m-2} b_{i}} \int_{\xi_{i}}^{T}\left(\frac{\sum_{i=1}^{m-2} a_{i} \int_{0}^{\xi_{i}} a(r) \nabla r}{1-\sum_{i=1}^{m-2} a_{i}}+\int_{0}^{\eta} a(r) \nabla r\right) \Delta s=133 / 48, \\
& Q=\frac{\sum_{i=1}^{m-2} a_{i} \int_{0}^{\xi_{i}} a(r) \nabla r}{1-\sum_{i=1}^{m-2} a_{i}}+\int_{0}^{T} a(r) \nabla r=11 / 4
\end{aligned}
$$

and

$$
\begin{aligned}
N= & \int_{0}^{T}\left(\frac{\sum_{i=1}^{m-2} a_{i} \int_{0}^{\xi_{i}} a(r) \nabla r}{1-\sum_{i=1}^{m-2} a_{i}}+\int_{0}^{s} a(r) \nabla r\right) \Delta s \\
& +\frac{\sum_{i=1}^{m-2} b_{i}}{1-\sum_{i=1}^{m-2} b_{i}} \int_{\xi_{i}}^{T}\left(\frac{\sum_{i=1}^{m-2} a_{i} \int_{0}^{\xi_{i}} a(r) \nabla r}{1-\sum_{i=1}^{m-2} a_{i}}+\int_{0}^{s} a(r) \nabla r\right) \Delta s \\
<\tilde{N} & =\int_{0}^{T}\left(\frac{\sum_{i=1}^{m-2} a_{i} \int_{0}^{\xi_{i}} a(r) \nabla r}{1-\sum_{i=1}^{m-2} a_{i}}+\int_{0}^{T} a(r) \nabla r\right) \Delta s \\
& +\frac{\sum_{i=1}^{m-2} b_{i}}{1-\sum_{i=1}^{m-2} b_{i}} \int_{\xi_{i}}^{T}\left(\frac{\sum_{i=1}^{m-2} a_{i} \int_{0}^{\xi_{i}} a(r) \nabla r}{1-\sum_{i=1}^{m-2} a_{i}}+\int_{0}^{T} a(r) \nabla r\right) \Delta s \\
= & 473 / 48 .
\end{aligned}
$$


As a result, $f(t, w, v)$ satisfies

$$
\begin{aligned}
& f(t, w, v) \leq \min \left\{\phi_{p}\left(r_{2} / \tilde{N}\right), \phi_{p}\left(l_{2} / Q\right)\right\} \approx 14.2071<\min \left\{\phi_{p}\left(r_{2} / N\right), \phi_{p}\left(l_{2} / Q\right)\right\}, \\
& \text { when } 0 \leq t \leq 2,0 \leq w \leq 140,-80 \leq v \leq 80 ; \\
& f(t, w, v)>\phi_{p}(b / M) \approx 0.7218, \quad \text { when } 1 \leq t \leq 2,2 \leq w \leq 4,-80 \leq v \leq 80 ; \\
& f(t, w, v)<\min \left\{\phi_{p}\left(r_{1} / \tilde{N}\right), \phi_{p}\left(l_{1} / Q\right)\right\} \approx 0.0126<\min \left\{\phi_{p}\left(r_{1} / N\right), \phi_{p}\left(l_{1} / Q\right)\right\}, \\
& \text { when } 0 \leq t \leq 2,0 \leq w \leq 1 / 8,-1 / 4 \leq v \leq 1 / 4 .
\end{aligned}
$$

Then all conditions of Theorem 3.1 hold. Therefore, the BVP (4.1) and (4.2) have at least three positive solutions $u_{1}, u_{2}$ and $u_{3}$ such that

$$
\begin{array}{ll}
\max _{t \in[0,2]_{\mathbb{T}}}\left\{u_{1}(t)\right\}<1 / 8, \quad \sup _{t \in[0, T]_{\mathbb{T}}}\left|u_{1}^{\Delta}(t)\right|<1 / 4 ; & \\
2<\min _{t \in[1,2]_{\mathbb{T}}}\left\{u_{2}(t)\right\} \leq \max _{t \in[0,2]_{\mathbb{T}}}\left\{u_{2}(t)\right\} \leq 140, & \sup _{t \in[0,2]_{\mathbb{T}}}\left|u_{2}^{\Delta}(t)\right| \leq 80 ; \\
\min _{t \in[1,2]_{\mathbb{T}}}\left\{u_{3}(t)\right\}<2, \quad \max _{t \in[0,2]_{\mathbb{T}}}\left\{u_{3}(t)\right\}<4, & \sup _{t \in[0,2]_{\mathbb{T}}}\left|u_{3}^{\Delta}(t)\right| \leq 80 .
\end{array}
$$

\section{Existence of double positive solutions to (1.5) and (1.6)}

Throughout the section, we assume that the following conditions are satisfied:

(H1) $0, T \in \mathbb{T}, 0<\xi_{1}<\xi_{2}<\cdots<\xi_{m-2}<\rho(T), a_{i} \in[0,+\infty)$ satisfy $0<\sum_{i=1}^{m-2} a_{i}<1$;

$(\mathrm{H} 2) q(t) \in C_{\mathrm{ld}}\left([0, T]_{\mathbb{T}},[0,+\infty)\right)$ with $0<\int_{0}^{\xi_{i}} q(r) \nabla r<\infty$;

(H3) $f:[0, T]_{\mathbb{T}} \times[0,+\infty) \rightarrow(-\infty,+\infty)$ is continuous;

(H4) $q(t) f(t, u(t)) \not \equiv 0, f(t, 0) \geq 0$ on $[0, T]_{\mathbb{T}}$.

Lemma 5.1 If $h \in C_{\mathrm{ld}}[0, T]$, then

$$
\begin{aligned}
& \left(\phi\left(u^{\Delta}(t)\right)\right)^{\nabla}+h(t)=0, \quad t \in(0, T)_{\mathbb{T}} \\
& \phi\left(u^{\Delta}(0)\right)=0, \quad u(T)=\sum_{i=1}^{m-2} a_{i} u\left(\xi_{i}\right)
\end{aligned}
$$

has the unique solution

$$
\begin{aligned}
u(t)= & -\int_{0}^{t} \phi^{-1}\left(\int_{0}^{s} h(r) \nabla r\right) \Delta s \\
& +\frac{1}{1-\sum_{i=1}^{m-2} a_{i}}\left[-\sum_{i=1}^{m-2} a_{i} \int_{0}^{\xi_{i}} \phi^{-1}\left(\int_{0}^{s} h(r) \nabla r\right) \Delta s\right. \\
& \left.+\int_{0}^{T} \phi^{-1}\left(\int_{0}^{s} h(r) \nabla r\right) \Delta s\right]
\end{aligned}
$$

Lemma 5.2 Assume that (H1) holds, for $h \in C_{\mathrm{ld}}[0, T]$ and $h \geq 0$, then the unique solution $u$ of (5.1) and (5.2) satisfies

$$
u(t) \geq 0 \quad \text { for } t \in[0, T]_{\mathbb{T}} .
$$


Lemma 5.3 Assume that $(\mathrm{H} 1)$ holds, if $h \in C_{\mathrm{ld}}[0, T]$ and $h \geq 0$, then the unique solution $u$ of (5.1) and (5.2) satisfies

$$
\inf _{t \in[0, T]} u(t) \geq \gamma\|u\|
$$

where

$$
\gamma=\frac{\sum_{i=1}^{m-2} a_{i}\left(T-\xi_{i}\right)}{T-\sum_{i=1}^{m-2} a_{i} \xi_{i}}, \quad\|u\|=\max _{t \in[0, T]}|u(t)| .
$$

Let $E=C_{\mathrm{ld}}([0, T], R)$ be the set of all ld-continuous functions from $[0, T]$ to $R$, and let the norm on $C_{\mathrm{ld}}([0, T])$ be the maximum. Then the $C_{\mathrm{ld}}([0, T], R)$ is a Banach space. We define three cones by

$$
\begin{aligned}
& P=\left\{u: u \in E, u(t) \geq 0, \forall t \in[0, T]_{\mathbb{T}}\right\}, \\
& P^{\prime}=\left\{u: u \in E, u(t) \text { is nonnegative, concave and decreasing on }[0, T]_{\mathbb{T}}\right\}
\end{aligned}
$$

and

$$
K=\left\{u: u \in E, u(t) \text { is nonnegative and decreasing on }[0, T]_{\mathbb{T}}, \inf _{t \in[0, T]_{\mathbb{T}}} u(t) \geq \gamma\|u\|\right\} \text {, }
$$

where $\gamma$ is the same as in Lemma 5.3. It is easy to see that the BVP (1.5) and (1.6) has a solution $u=u(t)$ if and only if $u$ solves the equation

$$
\begin{aligned}
u(t)= & -\int_{0}^{t} \phi^{-1}\left(\int_{0}^{s} q(r) f(r, u(r)) \nabla r\right) \Delta s \\
& +\frac{1}{1-\sum_{i=1}^{m-2} a_{i}}\left[-\sum_{i=1}^{m-2} a_{i} \int_{0}^{\xi_{i}} \phi^{-1}\left(\int_{0}^{s} q(r) f(r, u(r)) \nabla r\right) \Delta s\right. \\
& \left.+\int_{0}^{T} \phi^{-1}\left(\int_{0}^{s} q(r) f(r, u(r)) \nabla r\right) \Delta s\right] .
\end{aligned}
$$

We define the operators $A: P \rightarrow E, T: P^{\prime} \rightarrow E$ and $S: K \rightarrow E$ as follows:

$$
\begin{aligned}
(A u)(t)= & -\int_{0}^{t} \phi^{-1}\left(\int_{0}^{s} q(r) f(r, u(r)) \nabla r\right) \Delta s \\
& +\frac{1}{1-\sum_{i=1}^{m-2} a_{i}}\left[-\sum_{i=1}^{m-2} a_{i} \int_{0}^{\xi_{i}} \phi^{-1}\left(\int_{0}^{s} q(r) f(r, u(r)) \nabla r\right) \Delta s\right. \\
& \left.+\int_{0}^{T} \phi^{-1}\left(\int_{0}^{s} q(r) f(r, u(r)) \nabla r\right) \Delta s\right], \\
(T u)(t)= & \left(-\int_{0}^{t} \phi^{-1}\left(\int_{0}^{s} q(r) f(r, u(r)) \nabla r\right) \Delta s\right. \\
& +\frac{1}{1-\sum_{i=1}^{m-2} a_{i}}\left[-\sum_{i=1}^{m-2} a_{i} \int_{0}^{\xi_{i}} \phi^{-1}\left(\int_{0}^{s} q(r) f(r, u(r)) \nabla r\right) \Delta s\right.
\end{aligned}
$$




$$
\begin{aligned}
& \left.\left.+\int_{0}^{T} \phi^{-1}\left(\int_{0}^{s} q(r) f(r, u(r)) \nabla r\right) \Delta s\right]\right)^{+}, \\
(S u)(t)= & -\int_{0}^{t} \phi^{-1}\left(\int_{0}^{s} q(r) f^{+}(r, u(r)) \nabla r\right) \Delta s \\
& +\frac{1}{1-\sum_{i=1}^{m-2} a_{i}}\left[-\sum_{i=1}^{m-2} a_{i} \int_{0}^{\xi_{i}} \phi^{-1}\left(\int_{0}^{s} q(r) f^{+}(r, u(r)) \nabla r\right) \Delta s\right. \\
& \left.+\int_{0}^{T} \phi^{-1}\left(\int_{0}^{s} q(r) f^{+}(r, u(r)) \nabla r\right) \Delta s\right],
\end{aligned}
$$

where

$$
(C)^{+}=\max \{C, 0\}, \quad f^{+}(t, u(t))=\max \{f(t, u(t)), 0\}, \quad t \in[0, T]_{\mathbb{T}} .
$$

Lemma 5.4 $S: K \rightarrow K$ is completely continuous.

Proof It is obvious that $K$ is a cone in $E$. By $f^{+} \geq 0$, we get

$$
\int_{0}^{s} q(r) f^{+}(r, u(r)) \nabla r \geq 0
$$

Consequently,

$$
\phi^{-1}\left(\int_{0}^{s} q(r) f^{+}(r, u(r)) \nabla r\right) \geq 0 .
$$

This together with (5.4) imply that $(S u)(t) \geq 0$ and $S u$ is decreasing on $[0, T]_{\mathbb{T}}$. From

$$
(S u)^{\Delta}(t)=-\phi^{-1}\left(\int_{0}^{t} q(r) f^{+}(r, u(r)) \nabla r\right) \geq 0
$$

we see that $(S u)^{\Delta}$ is decreasing on $[0, T]_{\mathbb{T}}$.

(i) If $t$ is a left-scattered point, we have

$$
(S u)^{\Delta \nabla}=\frac{(S u)^{\Delta}(\rho(t))-(S u)^{\Delta}(t)}{\rho(t)-t} \leq 0 .
$$

(ii) If $t$ is a left-dense point, we have

$$
(S u)^{\Delta \nabla}=\lim _{s \rightarrow t} \frac{(S u)^{\Delta}(t)-(S u)^{\Delta}(s)}{t-s} \leq 0 .
$$

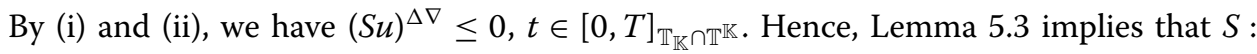
$K \rightarrow K$.

We now prove that $S$ is completely continuous.

(a) From the continuity of $f$ and $q(t) \in C_{\mathrm{ld}}\left([0, T]_{\mathbb{T}},[0,+\infty)\right)$, we find that $S$ is continuous. 
(b) Let $D$ be a bounded subset of $K$, and let $M>0$ be the constant such that $\|u\| \leq M$ for $u \in D$. The continuity of $f^{+}$guarantees that there is a $L>0$ such that

$$
\int_{0}^{s} q(r) f^{+}(r, u(r)) \nabla r \leq \int_{0}^{T} q(r) f^{+}(r, u(r)) \nabla r \leq \phi(L) .
$$

Thus,

$$
\begin{aligned}
\|S u\|= & \operatorname{Su}(0) \\
= & \frac{1}{1-\sum_{i=1}^{m-2} a_{i}}\left[-\sum_{i=1}^{m-2} a_{i} \int_{0}^{\xi_{i}} \phi^{-1}\left(\int_{0}^{s} q(r) f^{+}(r, u(r)) \nabla r\right) \Delta s\right. \\
& \left.+\int_{0}^{T} \phi^{-1}\left(\int_{0}^{s} q(r) f^{+}(r, u(r)) \nabla r\right) \Delta s\right] \\
\leq & \frac{\int_{0}^{T} \phi^{-1}\left(\int_{0}^{s} q(r) f^{+}(r, u(r)) \nabla r\right) \Delta s}{1-\sum_{i=1}^{m-2} a_{i}} \\
\leq & \frac{\int_{0}^{T} \phi^{-1}\left(\int_{0}^{T} q(r) f^{+}(r, u(r)) \nabla r\right) \Delta s}{1-\sum_{i=1}^{m-2} a_{i}} \leq \frac{L T}{1-\sum_{i=1}^{m-2} a_{i}} .
\end{aligned}
$$

(c) Let $t_{1}, t_{2} \in[0, T]_{\mathbb{T}}$, and let $u \in D$. Then we have

$$
\begin{aligned}
& \left|(S u)\left(t_{2}\right)-(S u)\left(t_{1}\right)\right| \\
& \quad=\left|-\int_{0}^{t_{2}} \phi^{-1}\left(\int_{0}^{s} q(r) f^{+}(r, u(r)) \nabla r\right) \Delta s+\int_{0}^{t_{1}} \phi^{-1}\left(\int_{0}^{s} q(r) f^{+}(r, u(r)) \nabla r\right) \Delta s\right| \\
& =\left|\int_{t_{2}}^{t_{1}} \phi^{-1}\left(\int_{0}^{s} q(r) f^{+}(r, u(r)) \nabla r\right) \Delta s\right| \leq L\left|t_{2}-t_{1}\right| .
\end{aligned}
$$

From (a)-(c) together with the Arzela-Ascoli theorem on time scales, we can find that $S: K \rightarrow K$ is completely continuous. This proof is complete.

The following fixed point index theory will play an important role in the proof our results.

Lemma $5.5([37,38])$ Let $E$ be a Banach space, and let $K$ be a cone in E. For $r>0$, define $K_{r}=\{u \in K:\|u\|<r\}$. Assume that $S: \bar{K}_{r} \rightarrow K$ is a completely continuous operator such that $T u \neq u$ for $u \in \partial K_{r}=\{u \in K:\|u\|=r\}$.

(a) If $\|T u\| \leq\|u\|$ for all $u \in \partial K_{r}$, then $i\left(T, K_{r}, K\right)=1$.

(b) If $\|T u\| \geq\|u\|$ for all $u \in \partial K_{r}$, then $i\left(T, K_{r}, K\right)=0$.

For notational convenience, we introduce the following notations. Let

$$
\begin{aligned}
& R=\phi^{-1}\left(\int_{0}^{T} q(r) \nabla r\right)\left(\frac{T-\sum_{i=1}^{m-2} a_{i} \xi_{i}}{1-\sum_{i=1}^{m-2} a_{i}}\right), \\
& Q=\frac{1}{1-\sum_{i=1}^{m-2} a_{i}}\left[\phi^{-1}\left(\int_{0}^{\xi_{i}} q(r) \nabla r\right) T-\sum_{i=1}^{m-2} a_{i} \phi^{-1}\left(\int_{0}^{\xi_{i}} q(r) \nabla r\right) \xi_{i}\right] .
\end{aligned}
$$


Theorem 5.1 Assume that conditions (H1), (H2), (H3) and (H4) are satisfied, there exist $b, c, d>0$ such that $0<\frac{d}{\gamma}<c<\gamma b<b$. Assume further that $f(t, u)$ satisfies the following conditions:

(i) $f(t, u) \geq 0, t \in[0, T]_{\mathbb{T}}, u \in[d, b]$;

(ii) $f(t, u)<\phi\left(\frac{c}{R}\right), t \in[0, T]_{\mathbb{T}}, u \in[0, c]$;

(iii) $f(t, u)>\phi\left(\frac{b}{Q}\right), t \in[0, T]_{\mathbb{T}}, u \in[\gamma b, b]$.

Then (1.5) and (1.6) has at least two positive solutions $u_{1}$ and $u_{2}$.

Proof We first verify that $T$ has a fixed point $u_{1} \in K$ with $c>\left\|u_{1}\right\|>0$. By condition (ii), we have for all $u(t) \in \partial K_{c}$,

$$
\int_{0}^{s} q(r) f(r, u(r)) \nabla r<\phi\left(\frac{c}{R}\right) \int_{0}^{s} q(r) \nabla r
$$

so that

$$
\phi^{-1}\left(\int_{0}^{s} q(r) f(r, u(r)) \nabla r\right)<\frac{c}{R} \phi^{-1}\left(\int_{0}^{s} q(r) \nabla r\right)<\frac{c}{R} \phi^{-1}\left(\int_{0}^{T} q(r) \nabla r\right) .
$$

Therefore,

$$
\begin{aligned}
\|T u\|= & \max _{t \in[0, T]_{\mathbb{T}}}\left(-\int_{0}^{t} \phi^{-1}\left(\int_{0}^{s} q(r) f^{+}(r, u(r)) \nabla r\right) \Delta s\right. \\
& +\frac{1}{1-\sum_{i=1}^{m-2} a_{i}}\left[-\sum_{i=1}^{m-2} a_{i} \int_{0}^{\xi_{i}} \phi^{-1}\left(\int_{0}^{s} q(r) f^{+}(r, u(r)) \nabla r\right) \Delta s\right. \\
& \left.\left.+\int_{0}^{T} \phi^{-1}\left(\int_{0}^{s} q(r) f^{+}(r, u(r)) \nabla r\right) \Delta s\right]\right)^{+} \\
= & \frac{1}{1-\sum_{i=1}^{m-2} a_{i}}\left[-\sum_{i=1}^{m-2} a_{i} \int_{0}^{\xi_{i}} \phi^{-1}\left(\int_{0}^{s} q(r) f^{+}(r, u(r)) \nabla r\right) \Delta s\right. \\
& \left.+\int_{0}^{T} \phi^{-1}\left(\int_{0}^{s} q(r) f^{+}(r, u(r)) \nabla r\right) \Delta s\right] \\
< & \frac{c}{R} \phi^{-1}\left(\int_{0}^{T} q(r) \nabla r\right)\left(\frac{T-\sum_{i=1}^{m-2} a_{i} \xi_{i}}{1-\sum_{i=1}^{m-2} a_{i}}\right) \\
= & c=\|u\| .
\end{aligned}
$$

This implies that $\|T u\|<\|u\|$ for $u(t) \in \partial K_{c}$. By Lemma 5.5(a), we have $i\left(T, K_{c}, K\right)=1$.

So, $T$ has a fixed point $u_{1}$ in $K_{c}$. By assumptions (H1), (H3) and (H4), we know that $u_{1}$ is also a fixed point of $A$ in $K_{c}$. As a result, $u_{1}$ is a solution of problem (1.5), (1.6).

Next, we verify that $A$ has another fixed point $u_{2}$ such that $c<\left\|u_{2}\right\| \leq b$. Using condition (ii), we have for $u(t) \in \partial P_{c}$

$$
\|S u\|<\frac{c}{R} \phi^{-1}\left(\int_{0}^{T} q(r) \nabla r\right)\left(\frac{T-\sum_{i=1}^{m-2} a_{i} \xi_{i}}{1-\sum_{i=1}^{m-2} a_{i}}\right)=c=\|u\| .
$$


For $t \in[0, T]_{\mathbb{T}}$, we have $\gamma b \leq u(t) \leq b$. By condition (iii), we find for $u(t) \in \partial P_{b}$

$$
\int_{0}^{s} q(r) f^{+}(r, u(r)) \nabla r>\phi\left(\frac{b}{Q}\right) \int_{0}^{s} q(r) \nabla r
$$

so that

$$
\phi^{-1}\left(\int_{0}^{s} q(r) f^{+}(r, u(r)) \nabla r\right)>\frac{b}{Q} \phi^{-1}\left(\int_{0}^{s} q(r) \nabla r\right) .
$$

Moreover, we get

$$
\begin{aligned}
\|S u\|= & (S u)(0) \\
= & \frac{1}{1-\sum_{i=1}^{m-2} a_{i}}\left[\int_{0}^{T} \phi^{-1}\left(\int_{0}^{s} q(r) f^{+}(r, u(r)) \nabla r\right) \Delta s\right. \\
& \left.-\sum_{i=1}^{m-2} a_{i} \int_{0}^{\xi_{i}} \phi^{-1}\left(\int_{0}^{s} q(r) f^{+}(r, u(r)) \nabla r\right) \Delta s\right] \\
> & \frac{\frac{b}{Q} \int_{0}^{T} \phi^{-1}\left(\int_{0}^{s} q(r) \nabla r\right) \Delta s-\frac{b}{Q} \sum_{i=1}^{m-2} a_{i} \int_{0}^{\xi_{i}} \phi^{-1}\left(\int_{0}^{s} q(r) \nabla r\right) \Delta s}{1-\sum_{i=1}^{m-2} a_{i}} \\
> & \frac{\frac{b}{Q} \phi^{-1}\left(\int_{0}^{\xi_{i}} q(r) \nabla r\right) T-\frac{b}{Q} \sum_{i=1}^{m-2} a_{i} \phi^{-1}\left(\int_{0}^{\xi_{i}} q(r) \nabla r\right) \xi_{i}}{1-\sum_{i=1}^{m-2} a_{i}} \\
= & b=\|u\| .
\end{aligned}
$$

It follows from Lemma 5.5 that $i\left(S, P_{c}, P\right)=1, i\left(S, P_{b}, P\right)=0$.

Thus, $i\left(S, P_{b} \backslash \bar{P}_{c}, P\right)=-1$. Hence, $S$ has a fixed point in $P_{b} \backslash \bar{P}_{c}$ such that $c<\left\|u_{2}\right\| \leq b$.

In the end, we show that $u_{2}$ is also a fixed point of $A$ in $P_{b} \backslash \bar{P}_{c}$. We only have to verify that $A u=S u, \forall u(t) \in\left(P_{b} \backslash \bar{P}_{c}\right) \cap\{u: S u=u\}$, and we get $u_{2}(0)=\left\|u_{2}\right\|>c$. By Lemma 5.3, we find that

$$
\inf _{t \in[0, T]_{\mathbb{T}}} u_{2}(t) \geq \gamma\left\|u_{2}\right\|>\gamma c>d
$$

Hence, $b \geq u_{2}(t) \geq d$ for $t \in[0, T]_{\mathbb{T}}$. Using condition (i), we understand that $f^{+}\left(t, u_{2}(t)\right)=$ $f\left(t, u_{2}(t)\right)$. This implies that $A u_{2}=S u_{2}=u_{2}$. It means that $u_{2}$ is a positive solution with $b \geq\left\|u_{2}\right\|>c$. This completes the proof.

Example 5.1 Let $\mathbb{T}=\left\{1-\left(\frac{1}{2}\right)^{\mathbb{N}_{0}}\right\} \cup\left\{\frac{1}{3}, 1\right\}$, where $\mathbb{N}_{0}$ denotes the set of all nonnegative integers. Take $a_{1}=\frac{1}{2}, \xi_{1}=\frac{1}{3}, T=1$ and $q(t) \equiv 1, t \in[0, T]_{\mathbb{T}}$. Consider the following BVP on time scales

$$
\begin{aligned}
& \left(\phi\left(u^{\Delta}(t)\right)\right)^{\nabla}+f(t, u(t))=0, \quad t \in[0, T]_{\mathbb{T}}, \\
& \phi\left(u^{\Delta}(0)\right)=0, \quad u(T)=\frac{1}{2} u\left(\frac{1}{3}\right),
\end{aligned}
$$


where

$$
\begin{aligned}
& \phi(u)=u, \\
& f(t, u):=f(u)= \begin{cases}-8 u^{2}+1 & \text { for } 0 \leq u \leq \frac{1}{2}, \\
4 u-3 & \text { for } \frac{1}{2} \leq u \leq 1, \\
\frac{17}{137}(u-1)^{2}+1 & \text { for } 1 \leq u \leq 5, \\
\left(27-\frac{17 \times 16}{137}\right)(u-5)^{2}+\frac{17 \times 16}{137}+1 & \text { for } 5 \leq u \leq 15, \\
-10(u-5)^{2}+\left(27-\frac{17 \times 16}{137}\right) 10^{2}+\frac{17 \times 16}{137}+1 & \text { for } u \geq 15 .\end{cases}
\end{aligned}
$$

By calculating, we find

$$
\begin{aligned}
& \gamma=\frac{\sum_{i=1}^{m-2} a_{i}\left(T-\xi_{i}\right)}{T-\sum_{i=1}^{m-2} a_{i} \xi_{i}}=\frac{a_{1}\left(1-\xi_{1}\right)}{1-a_{1} \xi_{1}}=\frac{\frac{1}{2}\left(1-\frac{1}{3}\right)}{1-\frac{1}{2} \cdot \frac{1}{3}}=\frac{2}{5}, \\
& R=\phi^{-1}\left(\int_{0}^{T} q(r) \nabla r\right)\left(\frac{T-\sum_{i=1}^{m-2} a_{i} \xi_{i}}{1-\sum_{i=1}^{m-2} a_{i}}\right)=\phi^{-1}\left(\int_{0}^{1} \nabla r\right)\left(\frac{1-a_{1} \xi_{1}}{1-a_{1}}\right)=\frac{5}{3}
\end{aligned}
$$

and

$$
\begin{aligned}
Q & =\frac{1}{1-\sum_{i=1}^{m-2} a_{i}}\left[\phi^{-1}\left(\int_{0}^{\xi_{i}} q(r) \nabla r\right) T-\sum_{i=1}^{m-2} a_{i} \phi^{-1}\left(\int_{0}^{\xi_{i}} q(r) \nabla r\right) \xi_{i}\right] \\
& =\frac{1}{1-a_{1}}\left[\phi^{-1}\left(\int_{0}^{\xi_{1}} \nabla r\right)-a_{1} \phi^{-1}\left(\int_{0}^{\xi_{1}} \nabla r\right) \xi_{1}\right]=\frac{5}{9} .
\end{aligned}
$$

Let $b=15, c=5, d=1$, then $0<\frac{d}{\gamma}=\frac{1}{\frac{2}{5}}=\frac{5}{2}<c=5<\gamma b=\frac{2}{5} \cdot 15=6<b=15$, and $f$ satisfies

(1) $f(t, u)=f(u) \geq 0, d=1 \leq u \leq b=15$;

(2) $f(t, u)=f(u)<\phi\left(\frac{c}{R}\right)=\frac{5}{5}=3,0 \leq u \leq c=5$;

(3) $f(t, u)=f(u)>\phi\left(\frac{b}{Q}\right)=\frac{15}{\frac{5}{9}}=27, \gamma b=6 \leq u \leq b=15$.

So all the conditions of Theorem 5.1 hold. Thus, by Theorem 5.1, problems (5.5) and (5.6) have at least two positive solutions.

\section{Competing interests}

The author declares that he has no competing interests.

\section{Authors' contributions}

The author carried out the proof of the theorem and approved the final manuscript.

\section{Acknowledgements}

The author is very grateful to Professor Ravi P Agarwal and Donal O'Regan for their important comments and suggestions in improving this manuscript. The project is supported by Abdullah Gul University Foundation of Turkey (Project No. 5).

Received: 12 March 2013 Accepted: 31 July 2013 Published: 7 August 2013

\section{References}

1. Hilger, S: Analysis on measure chains - a unified approach to continuous and discrete calculus. Results Math. 18, 18-56 (1990)

2. Agarwal, RP, Bohner, M: Basic calculus on time scales and its applications. Results Math. 35, 3-22 (1999)

3. Agarwal, RP, O'Regan, D: Nonlinear boundary value problems on time scales. Nonlinear Anal. 44, 527-535 (2001)

4. Agarwal, RP, Bohner, M, O'Regan, D, Peterson, A: Dynamic equations on time scales: a survey. J. Comput. Appl. Math. $141,1-26(2002)$ 
5. Sun, HR: Triple positive solutions for $p$-Laplacian $m$-point boundary value problem on time scales. Comput. Math Appl. 58, 1736-1741 (2009)

6. Sang, Y, Su, H: Several existence theorems of nonlinear $m$-point boundary value problem for $p$-Laplacian dynamic equations on time scales. J. Math. Anal. Appl. 340, 1012-1026 (2008)

7. Zhang, Y: A multiplicity result for a generalized Sturm-Liouville problem on time scales. J. Differ. Equ. Appl. 16, 963-974 (2010)

8. Anderson, DR, Avery, R, Henderson, J: Existence of solutions for a one dimensional $p$-Laplacian on time-scales. J. Differ. Equ. Appl. 10, 889-896 (2004)

9. Anderson, DR: Existence of solutions for a first-order $p$-Laplacian BVP on time scales. Nonlinear Anal. 69, 4521-4525 (2008)

10. Sun, HR, Li, WT: Existence theory for positive solutions to one-dimensional $p$-Laplacian boundary value problems on time scales. J. Differ. Equ. 240, 217-248 (2007)

11. Dogan, A, Graef, JR, Kong, L: Higher-order semipositone multi-point boundary value problems on time scales. Comput. Math. Appl. 60, 23-35 (2010)

12. Dogan, A, Graef, JR, Kong, L: Higher-order singular multi-point boundary value problems on time scales. Proc. Edinb. Math. Soc. 54, 345-361 (2011)

13. Graef, JR, Kong, L: First-order singular boundary value problems with $p$-Laplacian on time scales. J. Differ. Equ. Appl. $17,831-839(2011)$

14. Bai, ZB, Ge, WG: Existence of three positive solutions for some second-order boundary value problems. Comput. Math. Appl. 48, 699-707 (2004)

15. Yang, Y, Xiao, D: On existence of multiple positive solutions for $\phi$-Laplacian multipoint boundary value. Nonlinear Anal. 71, 4158-4166 (2009)

16. Wang, Y, Ge, W: Existence of triple positive solutions for multi-point boundary value problems with a one dimensional p-Laplacian. Comput. Math. Appl. 54, 793-807 (2007)

17. Ji, D, Yang, Y, Ge, W: Triple positive pseudo-symmetric solutions to a four-point boundary value problem with p-Laplacian. Appl. Math. Lett. 21, 268-274 (2008)

18. Wang, Y, Ge, W: Multiple positive solutions for multipoint boundary value problems with one-dimensional p-Laplacian. J. Math. Anal. Appl. 327, 1381-1395 (2007)

19. Bai, Z, Ge, W, Wang, Y: Multiplicity results for some second-order four-point boundary value problems. Nonlinear Anal. 60, 491-500 (2005)

20. Sun, B, Ge, W, Zhao, D: Three positive solutions for multipoint one-dimensional $p$-Laplacian boundary value problems with dependence on the first order derivative. Math. Comput. Model. 45, 1170-1178 (2007)

21. Bohner, M, Peterson, A: Dynamic Equations on Time Scales: An Introduction with Applications. Birkhäuser, Boston (2001)

22. Bohner, M, Peterson, A: Advances in Dynamic Equations on Time Scales. Birkhäuser, Boston (2003)

23. Sang, Y, Su, H, Xu, F: Positive solutions of nonlinear $m$-point BVP for an increasing homeomorphism and homomorphism with sign changing nonlinearity on time scales. Comput. Math. Appl. 58, 216-226 (2009)

24. Han, W, Jin, Z, Kang, S: Existence of positive solutions of nonlinear $m$-point BVP for an increasing homeomorphism and positive homomorphism on time scales. J. Comput. Appl. Math. 233, 188-196 (2009)

25. Luo, H: Positive solutions to singular multi-point dynamic eigenvalue problems with mixed derivatives. Nonlinear Anal. 70, 1679-1691 (2009)

26. Su, YH, Li, WT, Sun, HR: Positive solutions of singular $p$-Laplacian BVPs with sign changing nonlinearity on time scales. Math. Comput. Model. 48, 845-858 (2008)

27. Anderson, DR: Solutions to second order three-point problems on time scales. J. Differ. Equ. Appl. 8, 673-688 (2002)

28. Sun, JP, Li, WT: Existence and nonexistence of positive solutions for second-order time scale systems. Nonlinear Anal. 68, 3107-3114 (2008)

29. Sun, HR, Li, WT: Positive solutions for nonlinear three-point boundary value problems on time scales. J. Math. Anal. Appl. 299, 508-524 (2004)

30. Zhu, Y, Zhu, J: The multiple positive solutions for $p$-Laplacian multipoint BVP with sign changing nonlinearity on time scales. J. Math. Anal. Appl. 344, 616-626 (2008)

31. He, ZM: Double positive solutions of three-point boundary value problems for $p$-Laplacian dynamic equations on time scales. J. Comput. Appl. Math. 182, 304-315 (2005)

32. He, ZM: Triple positive solutions of boundary value problems for $p$-Laplacian dynamic equations on time scales. J. Math. Anal. Appl. 321, 911-920 (2006)

33. $\mathrm{Su}, \mathrm{H}$, Wang, B, Wei, Z: Positive solutions of four-point boundary value problems for four-order $p$-Laplacian dynamic equations on time scales. J. Differ. Equ. 78, 1-13 (2006)

34. Liang, SH, Zhang, JH: The existence of countably many positive solutions for nonlinear singular $m$-point boundary value problems on time scales. J. Comput. Appl. Math. 223, 291-303 (2009)

35. Ma, RY: Existence of solutions of nonlinear m-point boundary value problems. J. Math. Anal. Appl. 256, 556-567 (2001)

36. Wang, Y, Hou, C: Existence of multiple positive solutions for one-dimensional p-Laplacian. J. Math. Anal. Appl. 315 , 144-153 (2006)

37. Deimling, K: Nonlinear Functional Analysis. Springer, New York (1985)

38. Guo, D, Lakshmikantham, V: Nonlinear Problems in Abstract Cones. Academic Press, San Diego (1988)

doi:10.1186/1687-1847-2013-238

Cite this article as: Dogan: Existence of multiple positive solutions for $p$-Laplacian multipoint boundary value problems on time scales. Advances in Difference Equations 2013 2013:238. 